\title{
Cretaceous and Eocene tectono-thermal events determined in the Inner Western Carpathians orogenic front Infratatricum
}

\author{
Marián PUTIŠ ${ }^{1, *}$, Martin DANIŠíK², Pavol SIMAN³ , Ondrej NEMEC ${ }^{1}$, \\ Čestmír TOMEK ${ }^{3}$ and Peter RUŽIČKA ${ }^{1}$
}

1 Comenius University, Department of Mineralogy and Petrology, Faculty of Natural Sciences, Ilkovičova 6, 84215 Bratislava, Slovak Republic

2 Curtin University, John de Laeter Centre, The Institute of Geoscience Research (TIGeR), Perth, Western Australia, 6102, Australia

3 Earth Science Institute, Slovak Academy of Sciences, Dúbravská cesta 9, 84005 Bratislava, Slovak Republic

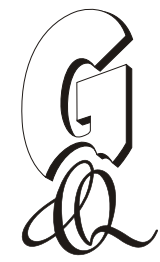

Putiš, M., Danišík, M., Siman, P., Nemec, O., Tomek, Č., Ružička, P., 2019. Cretaceous and Eocene tectono-thermal events determined in the Inner Western Carpathians orogenic front Infratatricum. Geological Quarterly, 63 (2): 248-274, doi: 10.7306/gq. 1467

Associate editor: Anna Wysocka

This paper re-assesses the tectono-thermal evolution of the frontal Infratatricum part of the Inner Western Carpathians orogen based on new geological-petrological and zircon fission track (ZFT) data and published ${ }^{40} \mathrm{Ar}-{ }^{39} \mathrm{Ar}$ and $\mathrm{K}-\mathrm{Ar}$ data. The study area is in the Považský Inovec Mts. in Western Slovakia, where the Infratatric Inovec Nappe comprises a micaschist-gneiss basement and Upper Carboniferous-Lower Cretaceous cover with the Jurassic-Lower Cretaceous Humienec Succession; this latter is reconstructed from olistoliths in the Horné Belice Succession Upper Cretaceous flysch. The Infratatric Inovec Nappe posterior part is thrust over its frontal Humienec tectonic Slice with infolded Upper Cretaceous sediments. This nappe exhibits very low-temperature tectono-thermal overprinting, and this is documented by electron probe microanalysis of metamorphic phases and P-T estimates. The late Early Cretaceous age of this D1 stage event at approximately $115-95 \mathrm{Ma}$ is recorded by $114 \pm 2$ and $106 \pm 4 \mathrm{Ma}$ phengitic white mica ${ }^{40} \mathrm{Ar}-{ }^{39} \mathrm{Ar}$ ages from a Lower Cretaceous cherty slate, and by $101+3 \mathrm{Ma}{ }^{40} \mathrm{Ar}-{ }^{39} \mathrm{Ar}$ age and $102+4 \mathrm{Ma}$ ZFT age from Permian meta-sandstone. The Inovec Nappe was thus derived from an Albian-Early Cenomanian accretionary wedge. The hemipelagic Upper Turonian to Lower Santonian red marls and upward syn-orogenic Upper Santonian to Maastrichtian flysch were deposited on the frontal attenuated part of the Inovec Nappe-type basement in the inferred Pieninic-Váhic ( South-Penninic) zone. This flysch contains olistolithic to clastogeneous metamorphosed material, including the Lower Cretaceous slates, supplied from the posterior part of the Inovec Nappe. The ${ }^{40} \mathrm{Ar}-{ }^{39} \mathrm{Ar}$ age of $86 \pm 2 \mathrm{Ma}$ from this nappes' Permian meta-sandstone is consistent with D2 thrusting at approximately 95-85 Ma and Horné Belice foreland flysch basin supply in the D3 stage at $\sim 85-65 \mathrm{Ma}$. The Inovec Nappe frontal Humienec tectonic Slice with infolded Upper Cretaceous sediments suggests formation of a Paleocene-Eocene accretionary wedge at approximately $65-40 \mathrm{Ma}$ in the D4 stage. This event is constrained by ${ }^{40} \mathrm{Ar}-{ }^{39} \mathrm{Ar}$ age of $48 \pm 2 \mathrm{Ma}$ from the Tatricum hanging wall blastomylonites and whole-rock $\mathrm{K}-\mathrm{Ar}$ age of $46 \pm 3 \mathrm{Ma}$ from a Permian basalt olistolith in the footwall Infratatricum Horné Belice flysch. The Infratatricum finally became part of an Eocene accretionary wedge north of the Hrádok-Zlatníky thrust-fault, and reheating and exhumation cooling is documented by 57-37 Ma ZFT ages. Although the Infratatricum exhibits Late Cretaceous and Eocene tectono-thermal imprints, it is a remnant of the Early Cretaceous structure at the Inner Western Carpathian front. This imposes the Infratatricum as a distal continental margin of the Penninicum.

Key words: Inner Western Carpathians, Infratatricum, orogenic wedge, tectono-thermal events, zircon fission track.

\section{INTRODUCTION}

INFRATATRICUM IN THE OROGENIC STRUCTURE OF THE INNER WESTERN CARPATHIANS

The Western Carpathians are an example of an orogenic system incorporating accretionary wedges (Platt, 1993; Brandon, 2004) formed due to closure of the Neo-Tethys

\footnotetext{
* Corresponding author, e-mail: marian.putis@uniba.sk
}

Received: August 22, 2018; accepted: February 15, 2019; first published online: April 26, 2019
Meliata and Atlantic Tethys Penninic oceanic and intra-continental basins and the related subduction-collision processes occurring between $\sim 150$ to $50 \mathrm{Ma}$ (as in fig. 10 in Putiš et al., 2009a; Kozur, 1991; Putiš, 1991a, b; Faryad, 1995; Dallmeyer et al., 1996; Plašienka et al., 1997; Neubauer et al., 2000; Schmid et al., 2008). The Western Carpathians are traditionally subdivided into three tectonic zones - the Outer, Central and Inner Western Carpathians (Plašienka et al., 1997). Here we prefer the division only into Outer and Inner Western Carpathians (OWC, IWC) in review of principal tectonic units.

The OWC are composed mostly of Cenozoic flysch deposits transformed into a system of $\mathrm{N}$-vergent rootless nappes incorporated in the large Magura and Silesian-Krosno units overriding the Neogene deposits of the North European Platform Carpathian Foredeep. 


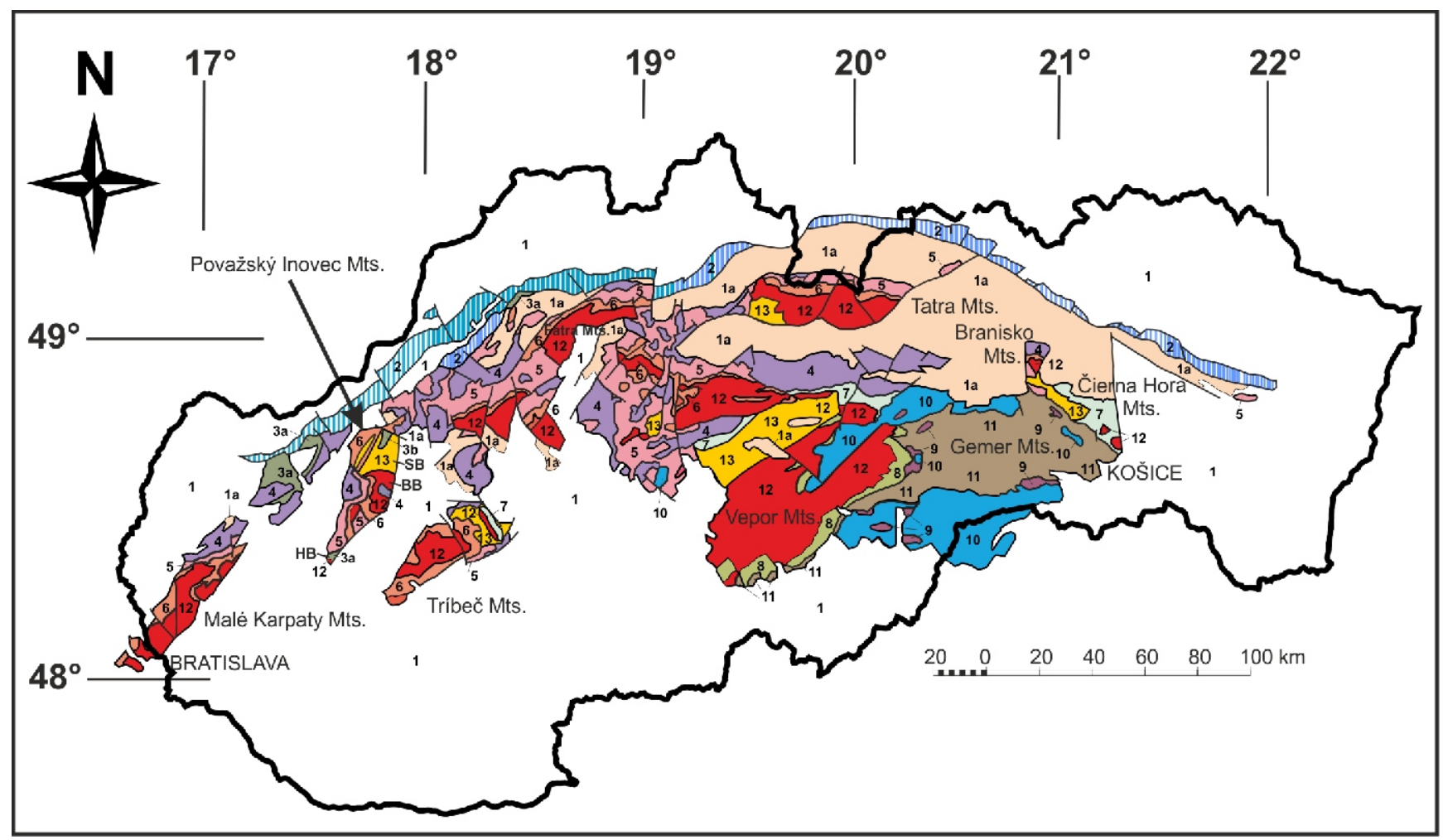

Fig. 1. The Inner Western Carpathians (IWC) sketch map (background from Lexa et al., 2000, modified)

1 - Quaternary and Cenozoic deposits undivided; 1a - Paleogene deposits of the IWC (south of PKB); 2 - Pieniny Klippen Belt (PKB) including the Manín and Klape units in western sector; 3a - Upper Cretaceous to Eocene Gosau-type sediments; $3 b$ - Upper Cretaceous (Infratatric, Horné Belice) Succession; 4 - Hronic nappes; 5 - Fatric (Krížna) nappes; 6 - Tatric cover; 7 - North-Veporic cover; 8 South-Veporic cover; 9 - Meliatic nappes; 10 - Silicic nappes; 11 - Gemeric Paleozoic basement (Variscan Lower Unit) and cover; 12 Variscan Upper Unit; 13 - Variscan Middle Unit; abbreviations: BB - Bojná Block ( Tatricum), HB - Hlohovec Block ( Infratatricum), SB Selec Block ( Infratatricum)

The Czorsztyn and Kysuca units of the Pieniny Klippen Belt (PKB), with unknown pre-Jurassic basement, mark the morphologically distinct Cenozoic tectonic boundary between the OWC Magura Unit ( North-Penninic) and the Klape, Drietoma, Manín and Infratatric Inovec nappe units of the IWC Peri-Pieniny Klippen Belt (PPKB), south of the inferred subducted Pieninic-Váhic ( South-Penninic) zone (see also Oszczypko et al., 2015 and references therein).

Southwards, a NW-vergent fold-and-thrust belt (Fig. 1) with crystalline basement and Upper Paleozoic to Mesozoic cover complexes ( Lower and Upper Austroalpine) is subdivided into the Tatric, Veporic and Gemeric units (Tatricum, Veporicum and Gemericum) that are separated by major Cretaceous shear zones (Putiš, 1991b; Tomek, 1993; Plašienka et al., 1997; Putiš et al., 1999). In general, the Tatric and Veporic Variscan basement consists mostly of the Variscan Upper (para- and ortho-gneisses, migmatites, amphibolites, rare eclogites and peridotites, intruded by S- and I-type granites to tonalites) and the Middle (monotonous micaschists to gneisses) units, while the Gemeric basement forms the Variscan Lower Unit in the south-vergent Variscan structure (Putiš, 1992; Bezák, 1994; Putiš et al., 2009b, 2018a). The Tatricum is overthrust by the Mesozoic Fatric nappes overlying the Cenomanian and in places also Turonian to Santonian(?) cover sediments (Pelech et al., 2017a and references therein) which in turn are overlain by the Hronic nappes. Finally, the Gemericum is overthrust by the Meliatic and higher Turnaic and Silicic nappes.
The Late Jurassic-Early Cretaceous Neotethyan Meliatic-South-Veporic-Gemeric accretionary wedge (Putiš et al., 2009a, 2014; Vozárová et al., 2014 and references therein) was underplated by the North-Veporic Unit crustal sheet. This event initiated the closure of the intra-continental basins towards the north at $\sim 115-95 \mathrm{Ma}$ and incorporation of the North-Veporic, Fatric and Tatric (including Infratatric) units in the late Early Cretaceous orogenic wedge (Putiš et al., 2009a). Consequently, the Albian-Lower Cenomanian syn-orogenic deep-marine terrigenous clastic Poruba and Klape-type flysches formed, which terminate the Jurassic-Lower Cretaceous sedimentary successions (Mišík et al., 1981; Mišík and Marschalko, 1988; Aubrecht et al., 2009).

Putiš (1992) introduced the "Infratatricum" term for the northern-most and lower-most structural complex of the Tatricum, previously called the Carpathian Penninicum by Leško et al. (1988), because of its position north of the typical Tatricum which was considered by Tollmann (1980) and Mahel' (1981) as an equivalent to the Lower Austroalpine of the Eastern Alps. Accordingly, the Infratatricum was recently reported as a distal Tatric continental margin facing northward to the South-Penninic Ocean (Putiš et al., 2006, 2008), while Plašienka et al. (1994) considered the Middle Jurassic to Upper Cretaceous Belice Succession representative of the oceanic Váhicum. Mahel' (1981) introduced the "Váhicum" term for the oceanic crust sources of the Klape Unit flysch conglomerates, supposedly representing a South-Penninic equivalent in the Western Carpathians. However, the blueschist pebbles 


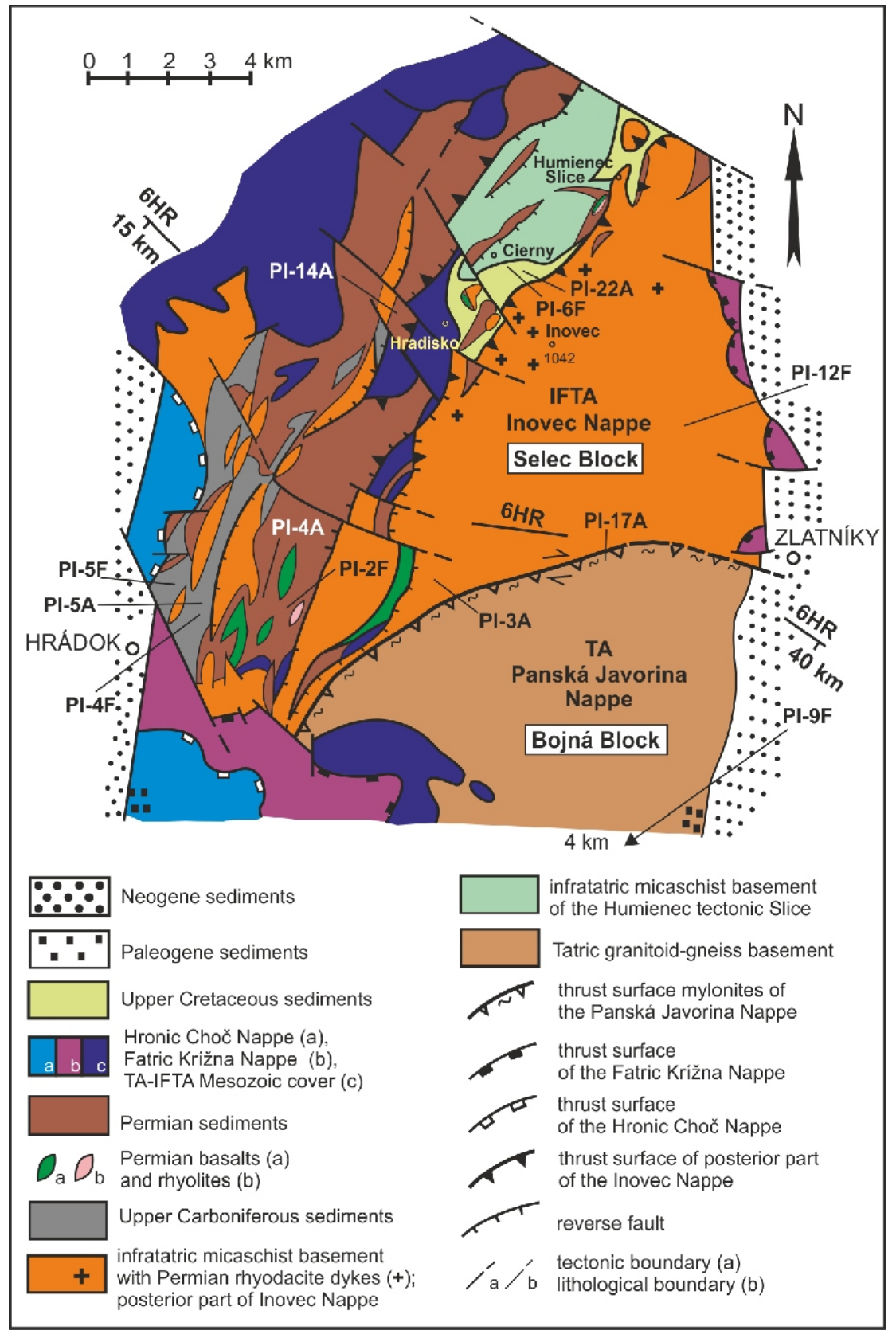

Fig. 2. Geological sketch-map of the middle and northern part of the Považský Inovec Mts. (compiled from Putiš 1983-2018; higher Mesozoic nappes from Ivanička et al., 2007)

IFTA - Infratatricum, TA - Tatricum; sample location from ZFT study; sample numbers are designed by " $\mathrm{F}$ ", and sample numbers from ${ }^{40} \mathrm{Ar}-{ }^{39} \mathrm{Ar}$ dating are designed by "A" at the end of the sample number; $6 \mathrm{HR}$ - reflection seismic line

glaucophane was dated at $\sim 155 \mathrm{Ma}$ by ${ }^{40} \mathrm{Ar}-{ }^{39} \mathrm{Ar}$ (Dal Piaz et al., 1995), thus resembling rather Neotethyan Meliatic Bôrka Nappe-type sources (Šímová, 1985; Dallmeyer et al., 1996; Faryad, 1997; Faryad and Henjes-Kunst, 1997; Kissová et al., 2005; Ivan et al., 2006; Putiš et al., 2014). We use the Váhicum term for the still undetermined but inferred subducted/obducted fragments of the Pieninic-Váhic oceanic crust as an equivalent of the Southern Penninicum.

The Infratatric basement-cover Inovec Nappe (Putiš, 1983; Putiš et al., 2016 and references therein), the PPKB Manín and Klape units, and the PKB Kysuca and Czorsztyn units all con- tain Upper Cretaceous shallow- to deep-water successions; mostly from Turonian upwards (Plašienka et al., 1997; Aubrecht et al., 2009; Plašienka and Soták, 2015; Oszczypko et al., 2015 and references therein).

This paper targets the Infratatricum, because it provides important clues to the tectonic evolution of the outer parts of the IWC, although it is exposed on the surface only in the Považský Inovec Mts. (Fig. 2) and resembles a Miocene tectonic window (Danišík et al., 2004; Putiš et al., 2008, 2018b). Moreover, the crosscutting NW-SE-directed reflection seismic profile $6 \mathrm{HR}$ (Figs. 2 and 3; see also Vozár et al., 1998; Vozár and Šantavý, 


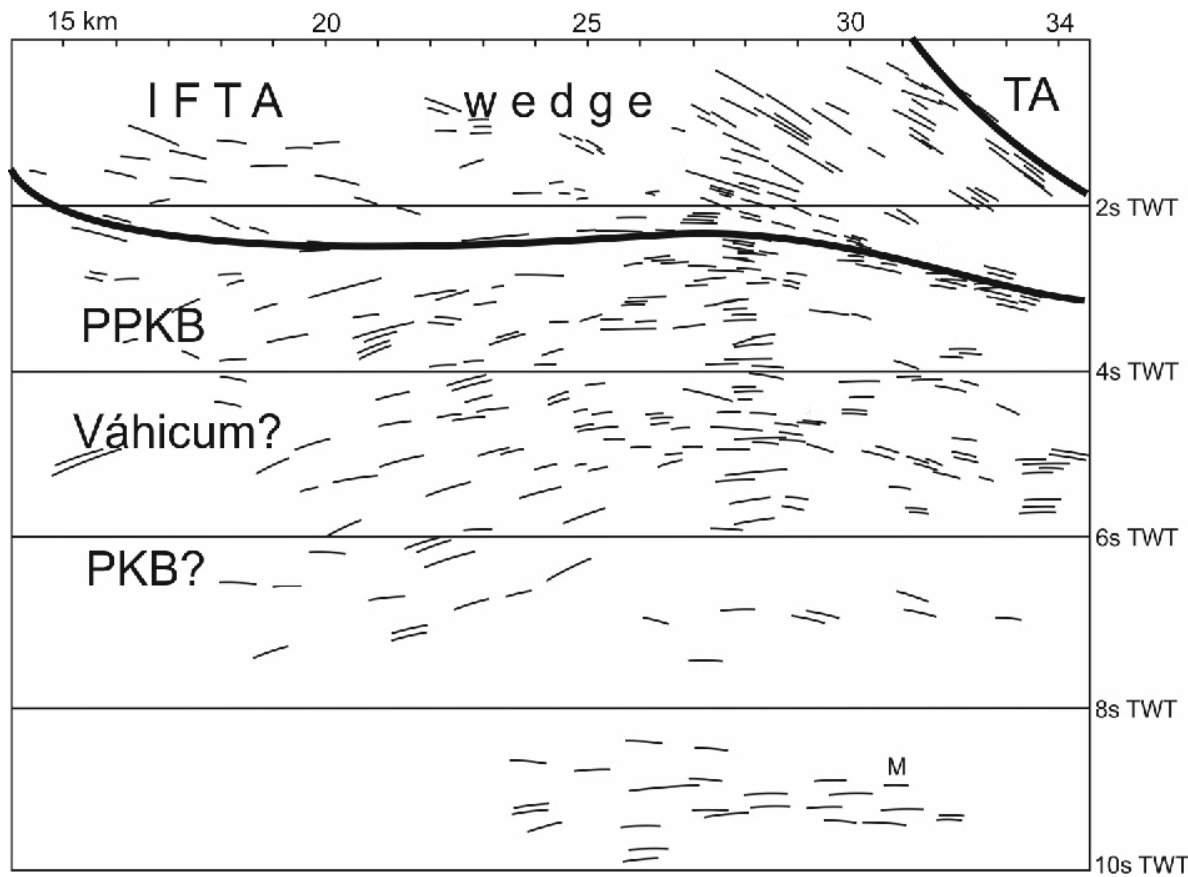

Fig. 3. Simplified reflection seismic profile 6HR (data from Vozár and Šantavý, 1999, processed by Č. Tomek) crosscutting the Považský Inovec Mts. in the NW-SE direction

IFTA - Infratatricum, PKB - Pieniny Klippen Belt, PPKB - Peri-Pieniny Klippen Belt, TA - Tatricum

1999) highlights an approximately a 6-7 km thick Infratatric accretionary wedge underthrust by a few crustal sheets recognizable above the $\mathrm{MOHO}$ boundary.

The Alpine tectono-metamorphic overprint of the Infratatricum remains under discussion. Králiková et al. (2016) concluded from two Permian zircon fission track (ZFT) ages that the Infratatricum in the Považský Inovec Mts. lacks an Alpine overprint. In contrast, Putiš (1983, 1986, 1992), Korikovsky et al. (1995), Putiš et al. (2006, 2008, 2009a, 2018b) and Sulák et al. (2009) all report petrological and geochronological data documenting distinct very low-temperature tectono-metamorphic overprinting.

This paper reviews the principal data on lithology and tectonic structures of the Považský Inovec Mts. New data on metamorphic overprinting grade estimates of the Infratatric Inovec Nappe and its metamorphosed olistolithic fragments in the Upper Cretaceous flysch sediments are combined with new ZFT and chosen published ${ }^{40} \mathrm{Ar}-{ }^{39} \mathrm{Ar}$ and $\mathrm{K}-\mathrm{Ar}$ data (Putiš et al., 2009a). This dataset provides a revised view on the Infratatricum. A special novelty is evidence that the Infratatricum was an active member of the Albian-Early Cenomanian accretionary wedge; with the subsequent Late Cretaceous and Eocene reactivation periods. This completely changes the previously proposed geological models recording a continuous "Infratatric" sedimentary cover succession up to the Late Cretaceous, without mentioning or disclaiming a late Early Cretaceous tectono-metamorphic event (as in Plašienka, 2018 and discussed models therein). Abbreviations for the names of rock-forming minerals in the text, tables and figures: Act actinolite, Ab-albite, Amph - amphibole, Ap - apatite (F-Ap fluorine-rich Ap), Bt, Bio - biotite, Cal - calcite, Cel - celadonite,
Chl - chlorite, Cpx - clinopyroxene, Czo - clinozoisite, Ep epidote, Gl-glaucophane, Hbl - hornblende, III - illite, III-Phg illite-phengite mixed-layer phase, Kfs - potassium feldspar, Law - lawsonite, Mag - magnetite, Ms - muscovite (Cel-Ms celadonite-rich Ms), Pg - pargasite, Phg - phengite (Cel-Ms), Pmp, Pu - pumpellyite, Qz - quartz, Ser - sericite (fine-flaked white mica), Sph - titanite, Stlp - stilpnomelane, $\mathrm{Tr}$ - tremolite, Ts - tschermakite.

\section{GEOLOGICAL REVIEW OF THE POVAŽSKÝ INOVEC MOUNTAINS}

The Považský Inovec Mts. form a Neogene horst in the northwestern part of the Western Carpathians. It is divided into the following three blocks: the northern Selec, the middle Bojná, and the southern Hlohovec (Figs. 1 and 2; Mahel', 1986). These blocks have numerous geological specifics. Only the Selec and Hlohovec blocks of the Považský Inovec Mts. have the PPKBand PKB-type Upper Cretaceous cover we use as the main criterion for determination of the Infratatricum.

\section{SELEC BLOCK (INFRATATRICUM)}

The tectonic boundary between the Selec and Bojná blocks is a transpressional thrust-fault (Fig. 2).

The Variscan crystalline basement of the Selec Block is formed by medium-grade micaschists to gneisses with rare amphibolite and orthogneiss bodies (Kamenický, 1956; Putiš, 1983, 1986; Krist et al., 1992; Ivanička et al., 2011). 
The Upper Paleozoic cover of the Selec Block consists of intra-continental Upper Carboniferous and Permian volcano-sedimentary rocks (Putiš, 1986; Olšavský, 2008; Putiš et al., 2016). The Permian siliciclastics contain rhyolite and basalt interlayers after the lava flows. Permian volcanics and siliciclastics also occur as large olistoliths in Upper Cretaceous flysch sediments. The meta-basalts were originally pyroxene-amphibole-spinel basalts to basaltic andesites (Putiš, 1986; Korikovsky et al., 1995; Putiš et al., 2006, 2008, 2016).

The Infratatric Mesozoic sedimentary succession (Plašienka et al., 1994; Putiš et al., 2006, 2008; Plašienka, 2012) differs from the Tatric sedimentary cover particularly by the thicker Upper Cretaceous sedimentary succession which developed after a late Early Cretaceous tectono-metamorphic event (Putiš et al., 2016; Putiš and Tomek, 2016).

The Lower Triassic quarzites, sandstones and conglomerates, together with the Middle to Upper(?) Triassic platformal carbonates, form part of the anchi-metamorphosed cover folded in micaschits to gneisses of the Infratatric Inovec Nappe.

The Jurassic to Lower Cretaceous members ( the Humienec Succession) were reconstructed solely from olistoliths in the Upper Cretaceous flysch (the Horné Belice Succession) which occurs exclusively in the frontal Humienec tectonic Slice of the Inovec Nappe. The Jurassic consists of quartzitic sandstones and sandy crinoidal limestones, upper Lower Jurassic reddish nodular limestones and wackestone re-sediments. This syn-rift stage is marked by olistostrome breccias passing into deep-water reddish nodular limestones with filaments. The mass-debris flow deposits are composed of sandstones with chaotically deposited detrital material of micaschists, Permian sedimentary and volcanic rocks, and Triassic to Lower Jurassic siliciclastics and carbonates with shallow-water micritic limestones, dolomites and brownish clasts of karstified carbonates. The clasts are embedded in a pelagic pseudo-matrix of reddish micritic carbonates or claystones, containing Involutina sp. foraminifera and ostracods, radiolarians, and ammonites (Putiš et al., 2008). In the late Middle Jurassic, carbonate sedimentation changed to cherty shales, radiolarites, rare chertified turbidites of shallow-water carbonates (Kimmeridgian to Tithonian?), a boudinaged layer of Maiolica-type limestone (Tithonian to Berriasian?), and clayey-cherty dark shales with Hedbergella foraminifera alternating with thin radiolarite layers (Lazy Formation; Plašienka et al., 1994).

Hemipelagic reddish marly shales and marly limestones of Couches Rouges-type (Lemoine, 2003) then followed in the Turonian to Lower Santonian. Turonian age is specified by foraminifera (Svinica Formation; Plašienka et al., 1994), and the stratigraphic extent of the Couches Rouges-type red marls was confirmed up to the Santonian by Marginotruncana coronata Bolli foraminifera by Putiš et al. (2006, 2008). Later, Rakús in Ivanička et al. (2011) re-defined the Jurassic to Upper Cretaceous Belice Succession of Plašienka et al. (1994) to the Upper Cretaceous Horné Belice Group composed of the flysch Hranty and Rázová Formations of former Belice Succession, leaving the Svinica Formation marlstones (Plašienka et al., 1994) as a separate Upper Cretaceous formation. Therefore, we use the Horné Belice Succession as an informal lithostratigraphic unit which also incorporates the Svinica Formation. Santonian to Maastrichtian flysch of the Horné Belice Succession has alternating fine-grained red-coloured clayey shales, yellow marly sandy shales, mm-thick marly shale laminae and interlayers of fine- to medium-grained brownish carbonatic sandstones, sandy limestones and conglomerates. The carbonatic shales and sandstones contain re-sedimented crinoids and radiolarite clasts.
Olistoliths, olistostromes and fault-related scarp breccias with cm-to-km rock-fragments form an integral part of the Upper Cretaceous flysch. These fragments include brecciated micaschists with a matrix of subangular rock-fragments $(0.3-5 \mathrm{~cm})$, with mineral clasts from the micaschists to gneisses, and Permian shales, sandstones, basalts and rhyolites. Permian siliciclastic sedimentary and volcanic rocks, Lower Triassic quartzites, Middle Triassic shallow-water carbonates, Upper(?) Triassic carbonates and carbonatic breccias, and upper Lower Jurassic to Lower Cretaceous pelagic sediments of the reconstructed Humienec Succession are also reported. Red-coloured patches of unlithified clayey to cherty pseudomatrix provide evidence for final deposition in a deep-water environment. The breccias in the prevailing "yellowish-greyish flysch" of the Čierny vrch Hill Beds (Rázová Formation) contain up to $0.5 \mathrm{~m}$ micaschist clasts, together with clasts of cherty slates and carbonatic sandstones. A polymict conglomerate horizon in the grey flysch turbidites formed in the relatively upper portion of the succession. However, Putiš et al. (2006) documented evidence of the Cenozoic deformation from the overturned flysch beds in the type-locality near the Čierny vrch Hill area (Fig. 2), and this agrees with the overturned succession of meta-radiolarites and dark cherty slates in olistoliths in the close neighbourhood. The lithological maps and profiles show numerous tectonic lenses in flysch, especially derived from olistolith-olistostrome bodies composed of the mutually refolded Upper Jurassic meta-radiolarites and the Lower Cretaceous dark cherty slates in flysch (Figs. 4-7). Part of less to practically unmetamorphosed material may be derived from the basement-cover rocks of the frontal Humienec tectonic Slice by southward shedding (Putiš et al., 2008).

Pelech et al. (2016a) consider this Upper Cretaceous type as Gosau-type wedge-top basin sediments with continuation to the Tatric cover Hubina Formation of the Bojná Block (Pelech et al., 2017a).

\section{TECTONIC STRUCTURES}

Complete geological mapping of the Považský Inovec Mts. was not available until the 1:50,000 regional map of Ivanička et al. (2007, 2011). The general 1:200,000 geological map from Kamenický in Buday et al. $(1962,1963)$ revealed the Selec and Bojná blocks separated by the inferred Alpine thrust-fault (Mahel', 1986). New structural-geological mapping at 1:25,000 by Putiš (Putiš, 1983, 1986) revealed complex basement-cover tectonic structures which were also outlined in the first geological profiles across the mountains (Putiš, 1983, 1986; Leško et al., 1988; Krist et al., 1992). Accordingly, partly preserved pre-Alpine structures of the Selec Block depict at least a two-stage Alpine overprint. Synforms, especially of the Permian rocks folded in the phyllonitized crystalline basement, are widespread in the Selec Block (Fig. 2) and superposition of the Alpine structures, indicating their polystadial evolution in the crystalline basement rocks and the Upper Paleozoic to Mesozoic cover, is best proven by the composite fold-thrust structures. The limbs of partly preserved large-scale to mesoscopic recumbent folds are gently inclined with E-W to NE-SW-trending axes and $15-30^{\circ}$ SE dipping metamorphic schistosity. The steepened parts of the limbs have SE dipping at $60-85^{\circ}$ and these document the superimposed fold-thrust, back-thrust to imbricate zones which may include Upper Cretaceous sediments.

Mesoscopic crenulation cleavage is characteristic in the Upper Cretaceous marly and flysch sediments (Putiš et al., 2008), while folds in the basement and Permian to Lower Cretaceous cover rocks, including those from olistoliths, are typically mani- 


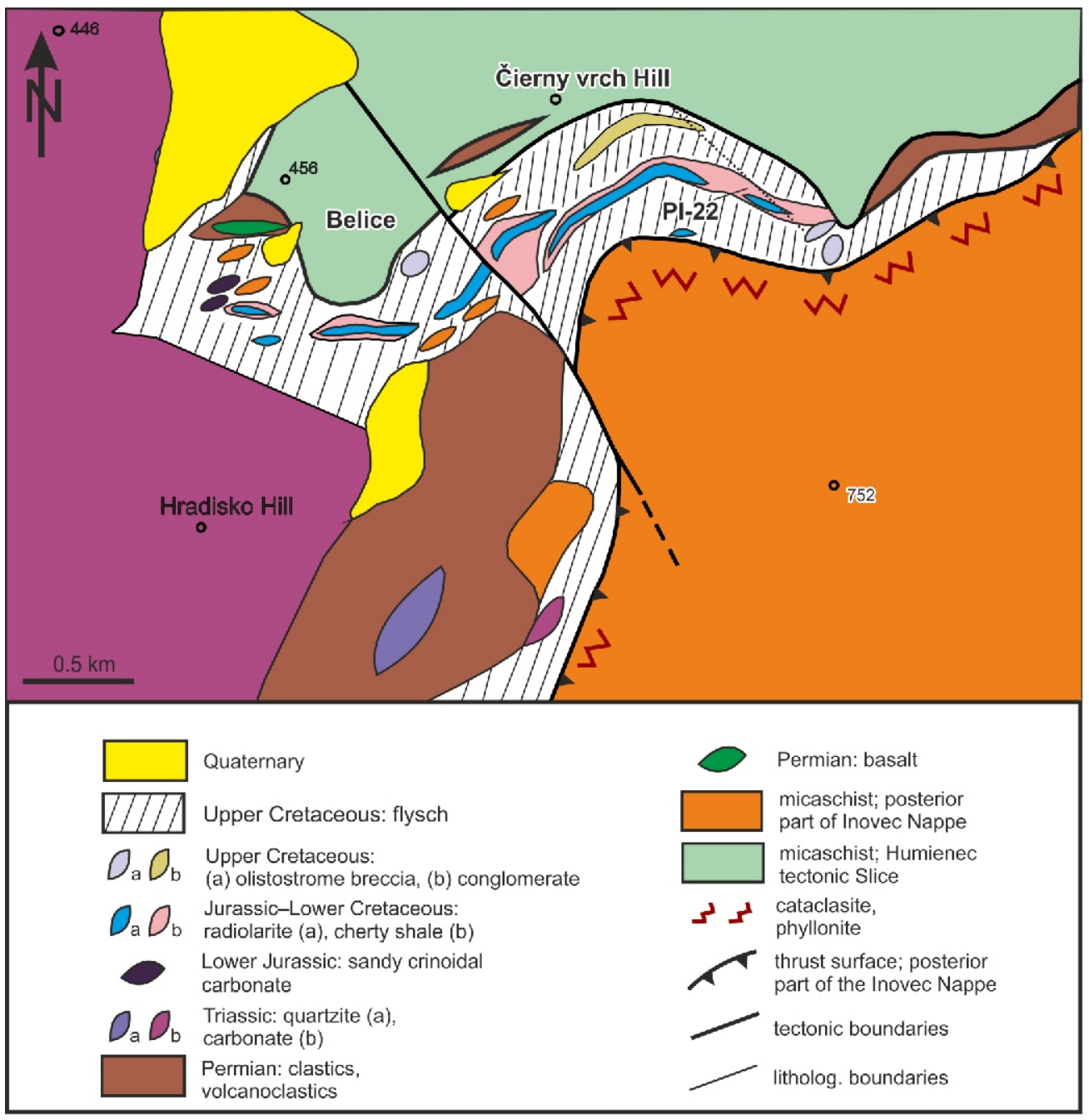

Fig. 4. Detailed map of the Upper Cretaceous flysch sediments with olistoliths of metamorphosed Permian to Lower Cretaceous rocks in the Čierny vrch Hill area (Putiš et al., 2008, modified)

Dotted profile line of lithological succession in Figure 5

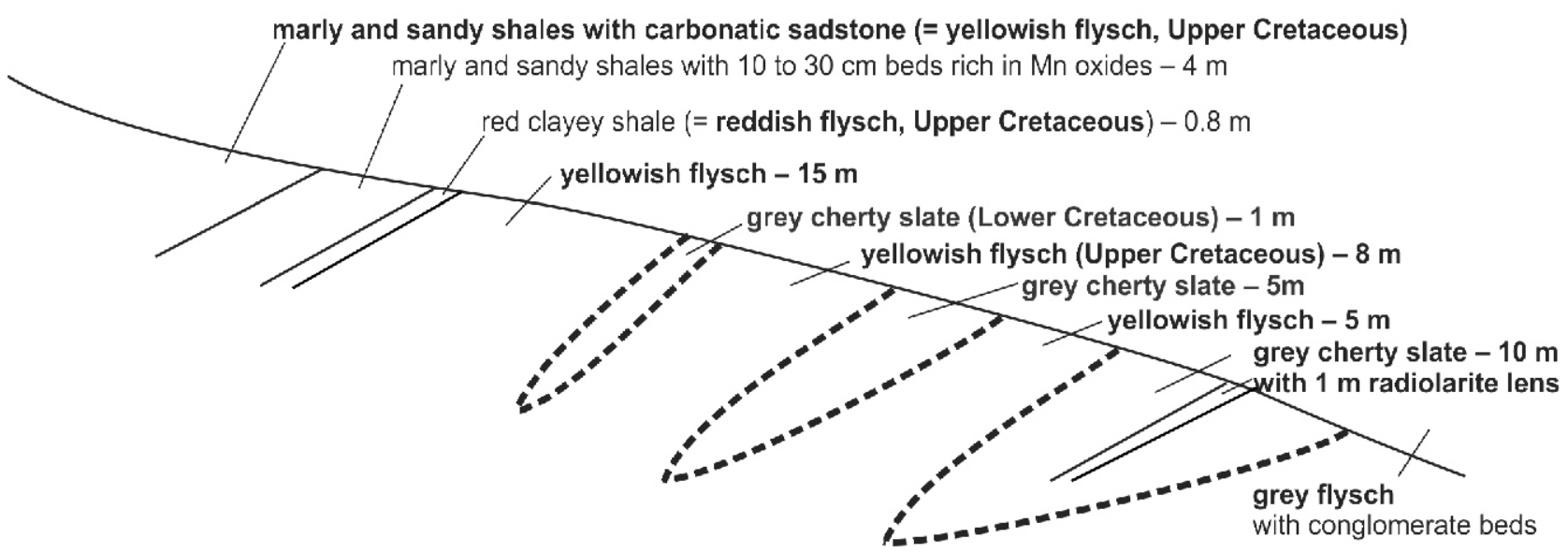

Fig. 5. Fragments (olistoliths originally) of Lower Cretaceous slates and Upper Jurassic meta-radiolarites in Upper Cretaceous flysch in the Čierny vrch Hill area 


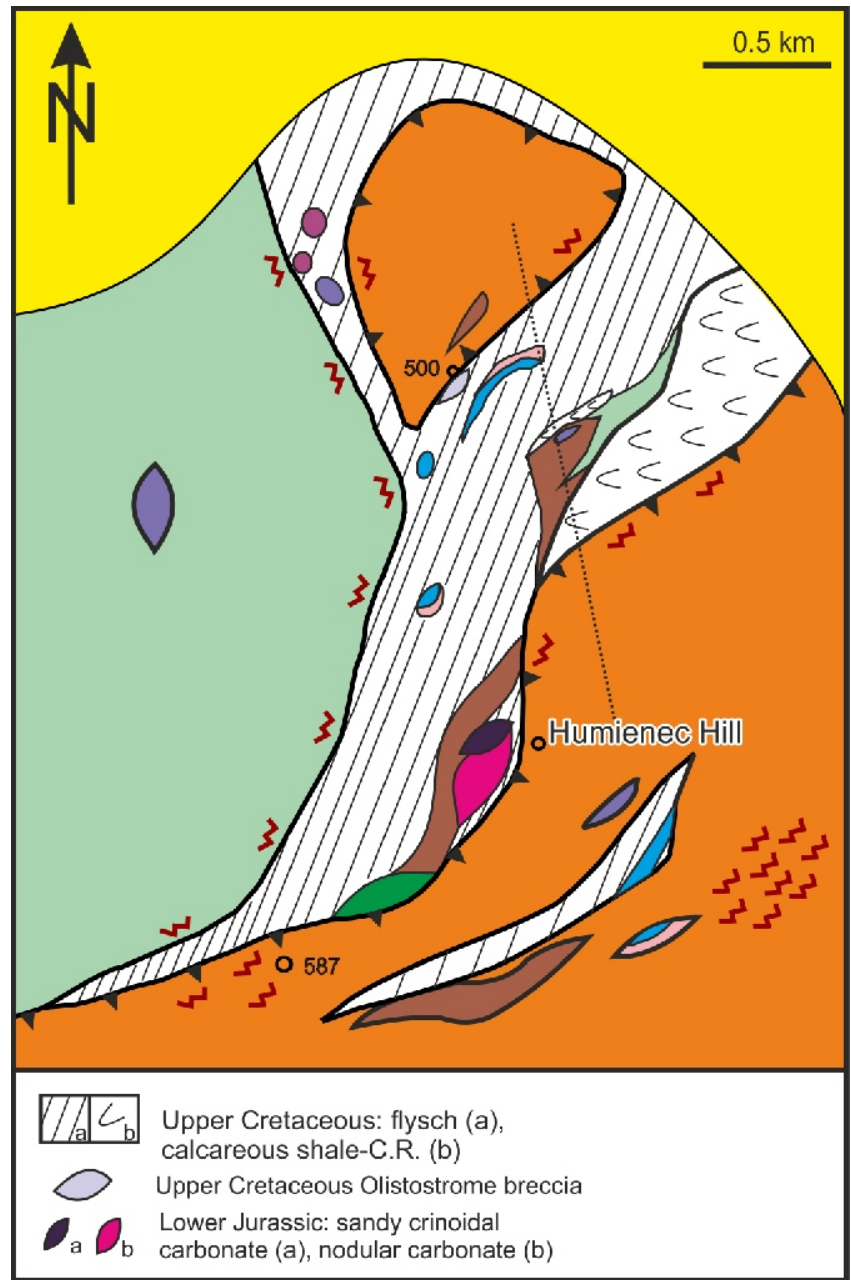

Fig. 6. Detailed map of the Upper Cretaceous flysch sediments with olistoliths of metamorphosed Permian to Lower Cretaceous rocks in the Humienec Hill area (Putiš et al., 2008, modified)

Dotted profile line of lithological succession in Figure 7; other explanations as in Figure 4 fested by isoclinal tight intrafolial folds with characteristic secondary transposition cleavage defined by the very low-grade metamorphic minerals (Putiš, 1986). Additional vast (micro-)structural data is available in published papers (Putiš, 1983, 1986; Putiš et al., 2006, 2008, 2009a).

Pelech et al. (2017b) established about 100 m infolded Upper Cretaceous sediments in the micaschist basement, while Plašienka et al. (1994) or Putiš et al. (2008) considered these exposures of Upper Cretaceous sediments as tectonic windows. The northern tectonic boundary of the Považský Inovec Mts. horst is formed by the generally NW-SE-striking Jastrabie fault (Mahel', 1986).

\section{BOJNÁ BLOCK (TATRICUM)}

The Bojná Block comprises the Tatric Panská Javorina Nappe (Putiš, 1983) overlying the Infratatric Inovec Nappe along the Hrádok-Zlatníky thrust-fault (Figs. 1 and 2). The Tatric block crystalline basement gneisses and amphibolites are intruded by large bodies of meso-Variscan granites covered by Triassic to Upper Cretaceous sedimentary successions (Mahel', 1986; Plašienka et al., 1997; Ivanička et al., 2011; Pelech et al., 2017a).

The pre-Alpine architecture is perfectly preserved in this block and an Alpine overprint is registered only by the Hrádok-Zlatníky thrust-fault zone blastomylonites of leucocrate granites (Putiš, 1983). This is a typical feature of the Tatricum and is also present in the neighbouring Malé Karpaty, Tribeč and Lúčanská Fatra Mts. where the newly formed blastomylonitic white micas were dated at $\sim 80-70 \mathrm{Ma}$ by ${ }^{40} \mathrm{Ar}-{ }^{39} \mathrm{Ar}$ ages from relatively thin deformation shear zones (Putiš, 1987, 1991b; Hók et al., 2000; Král' et al., 2002; Putiš et al., 2009a). The Hrádok-Zlatníky thrust-fault blastomylonites yielded $48 \pm 2 \mathrm{Ma}$ age, thus providing evidence for the Eocene reactivation of this inferred originally Cretaceous thrust-fault (Putiš et al., 2009a).

\section{HLOHOVEC BLOCK (INFRATATRICUM)}

The small Hlohovec Block is exposed on the surface at the southern termination of the Považský Inovec Mts. (Fig. 1) and it has a Lower Triassic to Lower Jurassic cover succession (Havrila and Vaškovský, 1983; Ivanička et al., 1998, 2007, 2011). This block also contains the Upper Cretaceous Couches-Rouges-type marls (Havrila, 1983 in Mahel', 1986;

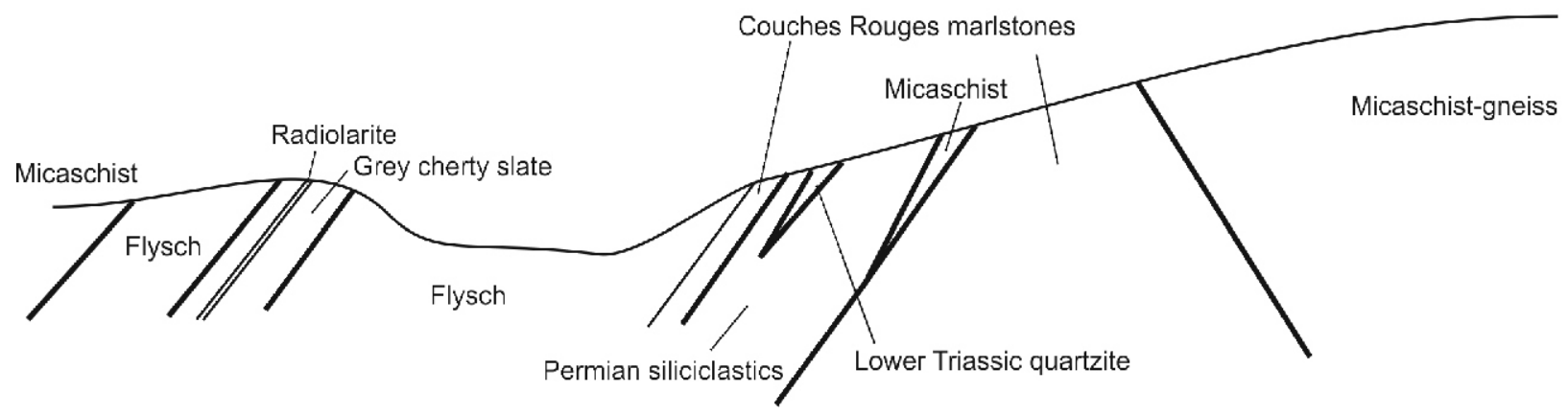

Fig. 7. Fragments (olistoliths originally) of metamorphosed Permian to Lower Cretaceous rocks in Upper Cretaceous flysch in the Humienec Hill area 


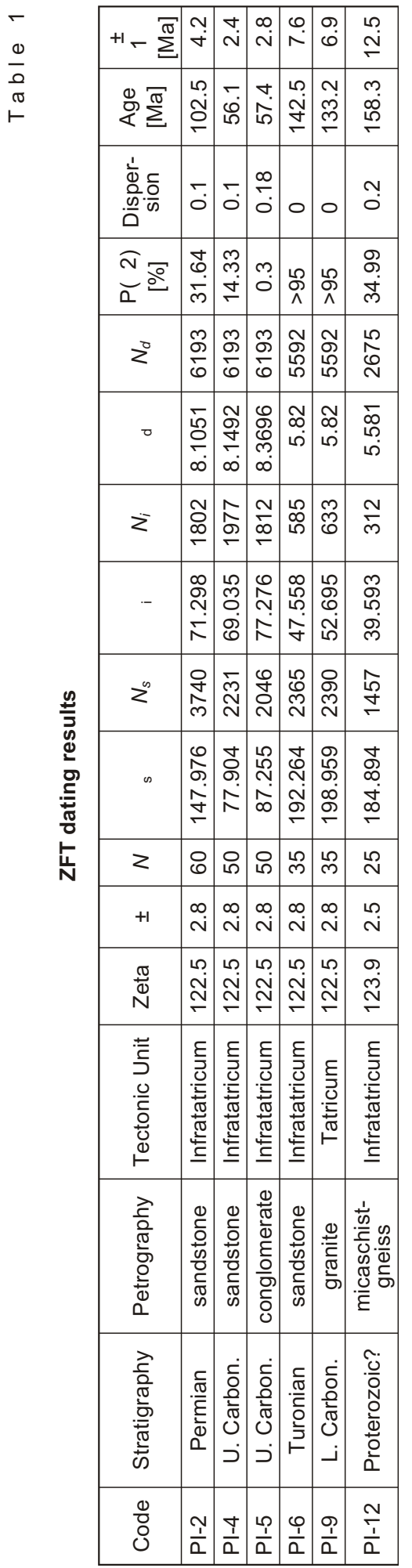

Ivanička et al., 2007, 2011) and flysch sediments (Pelech et al., 2016b) which are otherwise characteristic only for the Infratatricum area of the northern Selec Block, PPKB and PKB units. These sediments have a granitic basement (Fig. 1) and are overthrust mostly by the sheared Middle Triassic marbles (Havrila and Vaškovský, 1983; Putiš et al., 1999; Pelech et al., 2016b).

\section{SAMPLES AND METHODS}

The following six samples of crystalline basement and sedimentary rocks were analysed in this study; $\mathrm{PI}-2,4,5,6$ and 12 from the Selec Block and PI-9 from the Bojná Block of the Považský Inovec Mts. These were previously AFT dated (Danišík et al., 2004), and herein they are dated by ZFT to determine the presence, magnitude and timing of Alpine metamorphic events in the Infratatric and Tatric units (Tables 1 and 2; Fig. 8). The ZFT analysis followed Danišík et al. (2007), and Figure 2 illustrates the sample locations and geographic coordinates as in Danišík et al. (2005).

We adopted Gleadow's (1981) external detector method and Hurford and Green's (1983) $\xi$ age calibration approach to determine fission track (FT) age. Spontaneous tracks in zircon were revealed by etching in a eutectic mixture of $\mathrm{KOH}$ and $\mathrm{NaOH}$ at $215^{\circ} \mathrm{C}$ for 7 to 18 hours (Zaun and Wagner, 1985). Samples were analysed using a ZeissAxioskop 2 microscope (Carl Zeiss, Göttingen, Germany) with motorized x-y stage controlled by the FT Stage v. 3.11 computer program (Dumitru, 1993). Tracks were counted with $1250 \times$ magnification using a dry objective for mica detectors and an oil objective with Cargille oil type B for zircon $(n=1.515)$. Up to 60 grains per sample were analysed for age determination, depending on the availability of suitable zircon crystals and the distribution of single-grain ages, and FT ages were calculated by the TrackKey version $4.2 \mathrm{~g}$ program (Dunkl, 2002).

Several samples revealed complex single-grain age spectra with high dispersion. Here, the reported central FT age may not be entirely representative, so we identified sample individual age components by principal component analysis and PopShare software (Dunkl and Székely, 2003; http://www.sediment.uni-goettingen.de/staff/dunkl/software). This enables resolution of up to three components in mixed data using the Simplex algorithm for best-fit (Dantzig, 2016). Initially, we manually defined the principal age components by fitting peaks over distinct groupings in age spectrum plots to establish the best solution with the minimal root mean square value for "goodness of fit", and we then employed the Simplex algorithm with 500 interations to test their optimality.

The older electron probe micro-analyses (EPMA) of Korikovsky et al. (1995) were complemented with new EPMA of white micas, actinolite, epidote, chlorite, albite and phengite, together with magmatic relics of amphibole, clinopyroxene and spinel (Tables 3, 4, 5 and Figs. 9, 10). These were performed

ZFT age component analysis results

\begin{tabular}{|c|c|c|c|c|c|c|c|c|c|}
\hline \multirow[b]{2}{*}{$\begin{array}{l}\text { Sample } \\
\text { code }\end{array}$} & \multicolumn{3}{|c|}{ PC1 } & \multicolumn{3}{|c|}{ PC2 } & \multicolumn{3}{|c|}{ PC3 } \\
\hline & $\begin{array}{c}\text { Mean } \\
{[\mathrm{Ma}]}\end{array}$ & $\begin{array}{c}\text { Standard } \\
\text { deviation } \\
{[\mathrm{Ma}]}\end{array}$ & [\%] & $\begin{array}{c}\text { Mean } \\
{[\mathrm{Ma}]}\end{array}$ & $\begin{array}{c}\text { Standard } \\
\text { deviation } \\
{[\mathrm{Ma}]}\end{array}$ & [\%] & $\begin{array}{c}\text { Mean } \\
{[\mathrm{Ma}]}\end{array}$ & $\begin{array}{c}\text { Standard } \\
\text { deviation } \\
{[\mathrm{Ma}]}\end{array}$ & [\%] \\
\hline $\mathrm{PI}-2$ & 76.2 & 8.5 & 21 & 109.1 & 13.7 & 68 & 147.8 & 5.6 & 11 \\
\hline $\mathrm{PI}-4$ & 37.8 & 1.5 & 15 & 60.6 & 10.1 & 85 & & & \\
\hline $\mathrm{Pl}-5$ & 47.6 & 8.2 & 67 & 83.2 & 5.8 & 33 & & & \\
\hline $\mathrm{PI}-6$ & 142.5 & 32.6 & 100 & & & & & & \\
\hline $\mathrm{PI}-9$ & 133.2 & 16.1 & 100 & & & & & & \\
\hline $\mathrm{Pl}-12$ & 75 & 19.3 & 13 & 175.6 & 22.5 & 87 & & & \\
\hline
\end{tabular}



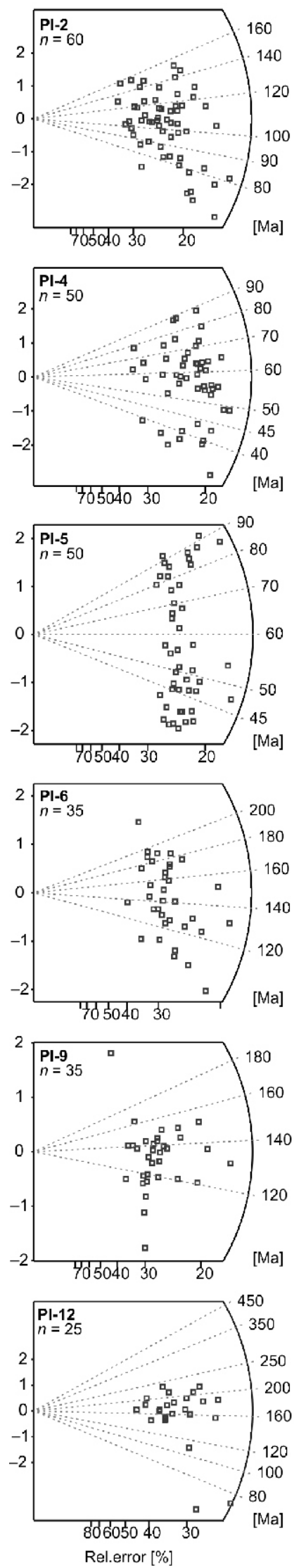
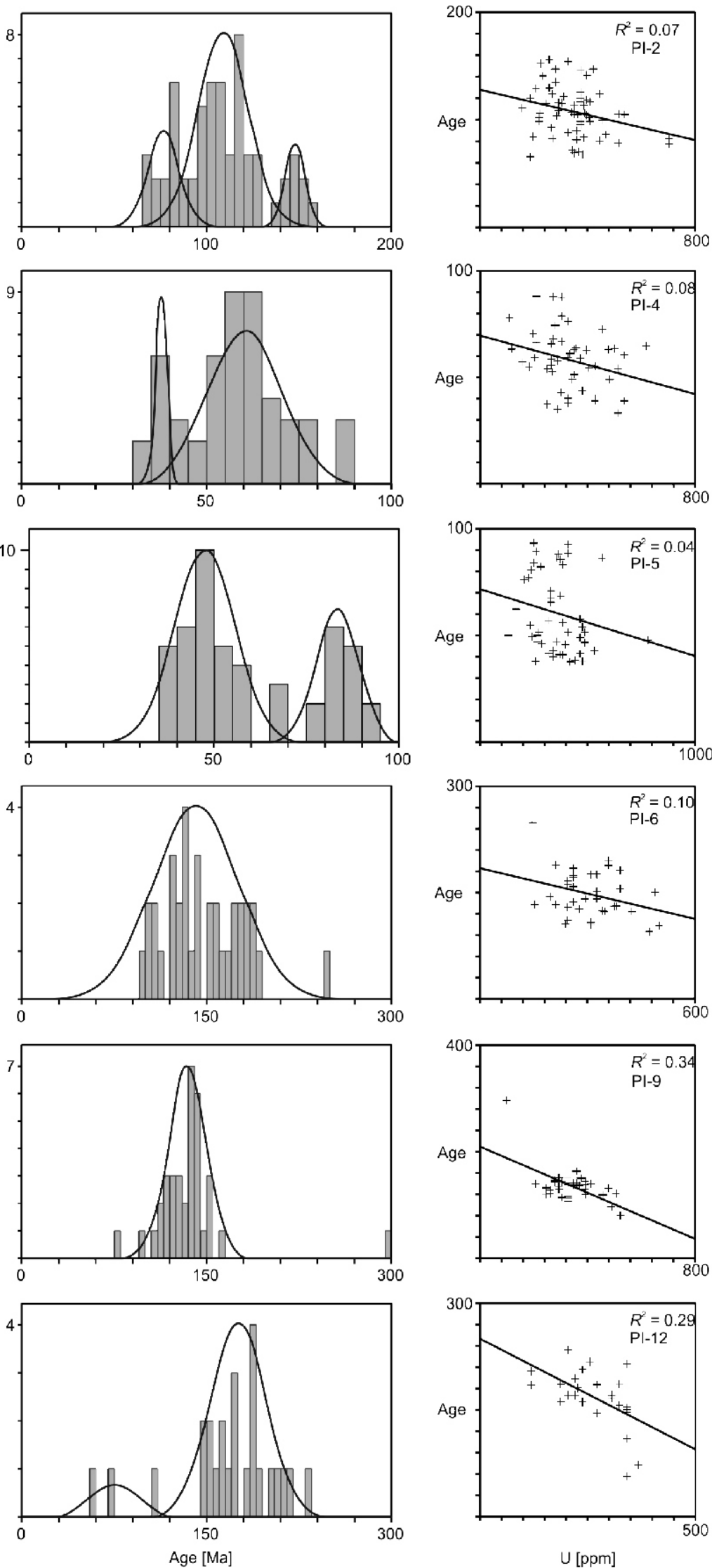

Fig. 8. Diagrams of single-grain ZFT ages displayed in radial plots, histograms with identifed major age components, and age vs. $U$ content plots

Explanation of diagrams: radial plots - left-hand-axis: $\sigma$ - error, bottom axis: relative error in \%, right-hand axis: age in Ma; histograms - y-axis: number of grains, $\mathrm{x}$-axis: age in $\mathrm{Ma}$ 
Representative EPMA of metamorphic albite, amphibole $\operatorname{Tr}($ Act) and epidote

\begin{tabular}{|c|c|c|c|c|c|c|c|c|c|}
\hline Sample & \multicolumn{4}{|c|}{ PI-BS1 } & \multicolumn{5}{|c|}{ PI-B3a } \\
\hline Rock age & \multicolumn{9}{|c|}{ Permian } \\
\hline Rock & \multicolumn{9}{|c|}{ Basalt } \\
\hline Mineral & $A b$ & & $\mathrm{Ep}$ & $\mathrm{Ep}$ & & \multicolumn{2}{|c|}{$\operatorname{Tr}($ Act $)$} & & $\mathrm{Cpx}$ \\
\hline \multicolumn{10}{|c|}{ [wt.\%] } \\
\hline $\mathrm{SiO}_{2}$ & 67.80 & $\mathrm{SiO}_{2}$ & 37.70 & 38.21 & $\mathrm{SiO}_{2}$ & 55.20 & 54.81 & $\mathrm{SiO}_{2}$ & 51.57 \\
\hline $\mathrm{TiO}_{2}$ & 0.00 & $\mathrm{TiO}_{2}$ & 0.53 & 0.04 & $\mathrm{TiO}_{2}$ & 0.00 & 0.09 & $\mathrm{TiO}_{2}$ & 1.00 \\
\hline $\mathrm{Al}_{2} \mathrm{O}_{3}$ & 21.08 & $\mathrm{Al}_{2} \mathrm{O}_{3}$ & 21.33 & 22.65 & $\mathrm{Al}_{2} \mathrm{O}_{3}$ & 0.74 & 1.18 & $\mathrm{Al}_{2} \mathrm{O}_{3}$ & 1.72 \\
\hline $\mathrm{Fe}_{2} \mathrm{O}_{3}$ & - & $\mathrm{Fe}_{2} \mathrm{O}_{3}$ & 13.13 & 13.43 & $\mathrm{Fe}_{2} \mathrm{O}_{3}$ & 0.05 & 1.21 & $\mathrm{Fe}_{2} \mathrm{O}_{3}$ & 0.62 \\
\hline $\mathrm{FeO}$ & 0.24 & $\mathrm{FeO}$ & 1.15 & 0.83 & $\mathrm{FeO}$ & 13.24 & 12.87 & $\mathrm{FeO}$ & 11.46 \\
\hline $\mathrm{MnO}$ & 0.00 & $\mathrm{MnO}$ & 0.04 & 0.33 & $\mathrm{MnO}$ & 0.30 & 0.33 & $\mathrm{MnO}$ & 0.35 \\
\hline $\mathrm{MgO}$ & 0.01 & $\mathrm{MgO}$ & 0.05 & 0.00 & $\mathrm{MgO}$ & 15.20 & 14.74 & $\mathrm{MgO}$ & 14.89 \\
\hline $\mathrm{CaO}$ & 0.08 & $\mathrm{CaO}$ & 23.02 & 23.03 & $\mathrm{CaO}$ & 12.45 & 11.85 & $\mathrm{CaO}$ & 18.12 \\
\hline $\mathrm{Na}_{2} \mathrm{O}$ & 11.55 & $\mathrm{Na}_{2} \mathrm{O}$ & 0.00 & 0.00 & $\mathrm{Na}_{2} \mathrm{O}$ & 0.29 & 0.62 & $\mathrm{Na}_{2} \mathrm{O}$ & 0.21 \\
\hline $\mathrm{K}_{2} \mathrm{O}$ & 0.04 & $\mathrm{~K}_{2} \mathrm{O}$ & 0.00 & 0.00 & $\mathrm{~K}_{2} \mathrm{O}$ & 0.06 & 0.08 & $\mathrm{~K}_{2} \mathrm{O}$ & 0.00 \\
\hline $\mathrm{H}_{2} \mathrm{O}^{*}$ & - & $\mathrm{H}_{2} \mathrm{O}^{*}$ & 1.85 & 1.89 & $\mathrm{H}_{2} \mathrm{O}^{*}$ & 1.80 & 1.80 & & - \\
\hline Total & 100.79 & Total & 98.80 & 100.40 & Total & 99.33 & 99.58 & Total & 99.99 \\
\hline \multicolumn{10}{|c|}{ Based on 5 cations - Ab, 3 Si and 8 cations $-E p, 13 e C N K-\operatorname{Tr}(a p f u), 4$ cations $-\mathrm{Cpx}$} \\
\hline $\mathrm{Si}^{4+}$ & 2.938 & $\mathrm{Si}^{4+}$ & 3.000 & 3.036 & $\mathrm{Si}^{4+}$ & 7.964 & 7.900 & $\mathrm{Si}^{4+}$ & 1.932 \\
\hline $\mathrm{Ti}^{4+}$ & 0.000 & $\mathrm{Ti}^{4+}$ & 0.032 & 0.002 & $\mathrm{Al}^{3+}$ & 0.036 & 0.100 & $\mathrm{Al}^{3+}$ & 0.068 \\
\hline $\mathrm{Al}^{3+}$ & 1.077 & $\mathrm{Al}^{3+}$ & 2.001 & 2.121 & $\Sigma T$ & 8.000 & 8.000 & $\Sigma T$ & 2.000 \\
\hline $\mathrm{Mg}^{2+}$ & 0.009 & $\mathrm{Fe}^{3+}$ & 0.786 & 0.803 & $\mathrm{Ti}^{4+}$ & 0.000 & 0.010 & $T i^{4+}$ & 0.028 \\
\hline $\mathrm{Mn}^{2+}$ & 0.000 & $\mathrm{Mn}^{2+}$ & 0.002 & 0.022 & $\mathrm{Al}^{3+}$ & 0.090 & 0.100 & $\mathrm{Al}^{3+}$ & 0.008 \\
\hline $\mathrm{Fe}^{2+}$ & 0.000 & $\mathrm{Fe}^{2+}$ & 0.077 & 0.055 & $\mathrm{Fe}^{3+}$ & 0.005 & 0.132 & $\mathrm{Fe}^{3+}$ & 0.018 \\
\hline $\mathrm{Ca}^{2+}$ & 0.004 & $\mathrm{Mg}^{2+}$ & 0.006 & 0.000 & $\mathrm{Mg}^{2+}$ & 3.270 & 3.167 & $\mathrm{Mg}^{2+}$ & 0.832 \\
\hline $\mathrm{Na}^{+}$ & 0.970 & $\Sigma M$ & 2.904 & 3.003 & $\mathrm{Mn}^{2+}$ & 0.037 & 0.041 & $\mathrm{Fe}^{2+}$ & 0.113 \\
\hline $\mathrm{K}^{+}$ & 0.002 & $\mathrm{Ca}^{2+}$ & 1.963 & 1.961 & $\mathrm{Fe}^{2+}$ & 1.598 & 1.551 & $\mathrm{Mn}^{2+}$ & 0.000 \\
\hline \multirow[t]{8}{*}{ Total } & 5.000 & $\mathrm{Na}^{+}$ & 0.000 & 0.000 & $\Sigma C$ & 5.000 & 5.000 & $\Sigma M 1$ & 1.000 \\
\hline & & $\mathrm{K}^{+}$ & 0.000 & 0.000 & $\mathrm{Ca}^{2+}$ & 1.925 & 1.830 & $\mathrm{Fe}^{2+}$ & 0.246 \\
\hline & & $\Sigma A$ & 1.963 & 1.961 & $\mathrm{Na}^{+}$ & 0.075 & 0.170 & $\mathrm{Mn}^{2+}$ & 0.011 \\
\hline & & $\mathrm{OH}^{-}$ & 1.000 & 1.000 & $\Sigma B$ & 2.000 & 2.000 & $\mathrm{Mg}^{2+}$ & 0.000 \\
\hline & & & & & $\mathrm{Na}^{+}$ & 0.006 & 0.004 & $\mathrm{Ca}^{2+}$ & 0.727 \\
\hline & & & & & $\mathrm{K}^{+}$ & 0.010 & 0.015 & $\mathrm{Na}^{+}$ & 0.016 \\
\hline & & & & & $\Sigma A$ & 0.016 & 0.019 & $\Sigma M 2$ & 1.000 \\
\hline & & & & & $\mathrm{OH}^{-}$ & 2.000 & 2.000 & Total & 4.000 \\
\hline
\end{tabular}

Classification of amphibole after Hawthorn et al. (2012); epidote after Armbruster et al. (2006)

by Cameca SX-100 at the State Geological Institute of D.S. in Bratislava. This study also includes chlorite thermometry (Cathelineau, 1988; Jowett, 1991), phengite barometry (Massonne and Schreyer, 1987) and Perple_X pseudo-section modelling to constrain P-T conditions of the late Early Cretaceous tectono-metamorphic overprint (D1 stage).

The P-T pseudo-sections were calculated by the Perple_ $X$ computer program package in $4-7 \mathrm{kbar}$ and $200-400^{\circ} \mathrm{C}$ ranges (Conolly and Petrini, 2002; Conolly, 2005). Calculations were performed using the thermodynamic dataset of Holland and Powell (1998, updated 2004). The following solid-solution models were chosen: $\mathrm{Chl}(\mathrm{HP}), \mathrm{Ep}(\mathrm{HP})$ for clinozoisite-epidote, Pheng(HP) for potassic white mica, Bio(HP) for biotite, $\mathrm{Pu}(\mathrm{M})$ for pumpellyite, GITrTsPg for amphiboles, and $\mathrm{Cpx}(\mathrm{HP})$ for clinopyroxene. However, no solid-solution model was selected for plagioclase because this phase is almost pure albite in very-low-grade metabasic rocks. The whole-rock composition of the PI-B3A sample was used $\left(\mathrm{SiO}_{2}=51.38, \mathrm{Al}_{2} \mathrm{O}_{3}=15.18\right.$,
$\mathrm{Fe}_{2} \mathrm{O}_{3}=10.76, \mathrm{MgO}=5.84, \mathrm{CaO}=6.96, \mathrm{Na}_{2} \mathrm{O}=2.72$, $\left.\mathrm{K}_{2} \mathrm{O}=1.34, \mathrm{TiO}_{2}=1.97, \mathrm{P}_{2} \mathrm{O}_{5}=0.41, \mathrm{MnO}=0.15\right)$. This was then calculated under the NCKFMASHTO system $\left(\mathrm{Na}_{2} \mathrm{O}-\mathrm{CaO}-\mathrm{K}_{2} \mathrm{O}-\mathrm{FeO}-\mathrm{MgO}-\mathrm{Al}_{2} \mathrm{O}_{3}-\mathrm{SiO}_{2}-\mathrm{H}_{2} \mathrm{O}-\mathrm{TiO}_{2}\right)$. The chemical composition of Phg and Chl (Tables 4 and 5 ) was used for isoplots and P-T conditions estimation (Fig. 11).

The rocks' original compositions were slightly modified to fit this 9-component system, as follows: (1) CaO was reduced according to bulk-rock phosphorous content, and assuming these elements are bound exclusively to ideally composed apatite, and (2) the $\mathrm{H}_{2} \mathrm{O}$ contents were set to 5 wt.\% to allow free hydrous fluid phase formation at relatively low P-T. The pseudo-sections were contoured with isoplets for various chemical parameters using Perple_ $X$ werami and pstable subprograms to supply raw data. The final pseudo-sections and countoured P-T diagrams were redrawn, and mineral assemblage data at specific $\mathrm{P}-\mathrm{T}$ conditions was supplied by Perple_ $X$ werami. 
Representative EPMA of white micas from the Permian rocks

\begin{tabular}{|c|c|c|c|c|c|c|c|c|c|c|c|}
\hline \multirow{3}{*}{$\begin{array}{c}\text { Sample } \\
\text { Rock age } \\
\text { Rock }\end{array}$} & $\mathrm{Pl}-2 \mathrm{~F}$ & PI-4A & \multicolumn{2}{|c|}{$\mathrm{PI}-14 \mathrm{~A}$} & \multicolumn{2}{|c|}{$\mathrm{Pl}-16$} & \multicolumn{2}{|c|}{ PI-B3a } & \multicolumn{2}{|c|}{ PI-R11b } & PI-RD1 \\
\hline & \multicolumn{11}{|c|}{ Permian } \\
\hline & \multicolumn{2}{|c|}{ Sandstone } & \multicolumn{2}{|c|}{ Sandstone } & \multicolumn{2}{|c|}{ Sandstone } & \multicolumn{2}{|c|}{ Basalt } & \multicolumn{2}{|c|}{ Rhyolite } & Rhyodacite \\
\hline Mineral & Cel-Ms & Cel-Ms & Ms clast & Cel-Ms & Cel-Ms & Cel-Ms & Cel-Ms & Cel-Ms & Cel-Ms & Cel-Ms & Cel-Ms \\
\hline \multicolumn{12}{|c|}{$[w t . \%]$} \\
\hline $\mathrm{SiO}_{2}$ & 48.78 & 50.05 & 48.88 & 51.31 & 47.63 & 47.37 & 49.43 & 49.95 & 51.50 & 52.18 & 48.44 \\
\hline $\mathrm{TiO}_{2}$ & 0.37 & 0.24 & 0.06 & 0.08 & 0.13 & 0.09 & 0.08 & 0.10 & 0.10 & 0.20 & 0.12 \\
\hline $\mathrm{Al}_{2} \mathrm{O}_{3}$ & 27.08 & 27.13 & 35.50 & 32.29 & 33.90 & 33.40 & 24.59 & 27.21 & 26.94 & 26.52 & 29.48 \\
\hline $\mathrm{FeO}$ & 5.58 & 4.86 & 0.74 & 1.03 & 3.24 & 3.45 & 6.08 & 5.06 & 3.51 & 3.18 & 3.12 \\
\hline $\mathrm{MnO}$ & 0.02 & 0.01 & 0.02 & 0.00 & 0.04 & 0.02 & 0.01 & 0.05 & 0.02 & 0.04 & 0.00 \\
\hline $\mathrm{MgO}$ & 1.92 & 2.06 & 0.72 & 0.82 & 0.80 & 0.85 & 2.94 & 2.26 & 2.75 & 2.88 & 1.99 \\
\hline $\mathrm{CaO}$ & 0.01 & 0.01 & 0.04 & 0.03 & 0.02 & 0.00 & 0.05 & 0.08 & 0.03 & 0.06 & 0.03 \\
\hline $\mathrm{Na}_{2} \mathrm{O}$ & 0.02 & 0.08 & 0.92 & 0.63 & 0.78 & 0.59 & 0.06 & 0.19 & 0.04 & 0.06 & 0.35 \\
\hline $\mathrm{K}_{2} \mathrm{O}$ & 11.04 & 10.86 & 9.02 & 9.45 & 8.92 & 9.56 & 10.81 & 10.75 & 10.49 & 9.57 & 9.71 \\
\hline $\mathrm{H}_{2} \mathrm{O}^{*}$ & 4.38 & 4.43 & 4.61 & 4.59 & 4.52 & 4.49 & 4.33 & 4.44 & 4.49 & 4.49 & 4.40 \\
\hline Total & 99.19 & 99.73 & 100.50 & 100.23 & 99.98 & 99.82 & 98.39 & 100.08 & 99.87 & 99.17 & 97.63 \\
\hline \multicolumn{12}{|c|}{ Based on 11 oxygen (apfu) } \\
\hline $\mathrm{Si}^{4+}$ & 3.341 & 3.386 & 3.182 & 3.348 & 3.160 & 3.162 & 3.421 & 3.373 & 3.442 & 3.484 & 3.303 \\
\hline${ }^{\mathrm{IV}} \mathrm{Al}^{3+}$ & 0.659 & 0.614 & 0.818 & 0.652 & 0.840 & 0.838 & 0.579 & 0.627 & 0.558 & 0.516 & 0.697 \\
\hline$\Sigma T$ & 4.000 & 4.000 & 4.000 & 4.000 & 4.000 & 4.000 & 4.000 & 4.000 & 4.000 & 4.000 & 4.000 \\
\hline $\mathrm{Mg}^{2+}$ & 0.062 & 0.045 & 0.019 & 0.000 & 0.079 & 0.073 & 0.087 & 0.059 & 0.040 & 0.047 & 0.059 \\
\hline$\square$ & 0.938 & 0.955 & 0.981 & 1.000 & 0.921 & 0.927 & 0.913 & 0.941 & 0.960 & 0.953 & 0.941 \\
\hline $\mathrm{Ti}^{4+}$ & 0.019 & 0.012 & 0.003 & 0.004 & 0.007 & 0.005 & 0.004 & 0.005 & 0.005 & 0.010 & 0.006 \\
\hline${ }^{\mathrm{V} \mid} \mathrm{Al}^{3+}$ & 1.527 & 1.550 & 1.905 & 1.831 & 1.811 & 1.790 & 1.427 & 1.538 & 1.564 & 1.571 & 1.673 \\
\hline $\mathrm{Fe}^{2+}$ & 0.320 & 0.275 & 0.040 & 0.056 & 0.180 & 0.192 & 0.352 & 0.286 & 0.196 & 0.177 & 0.178 \\
\hline $\mathrm{Mn}^{2+}$ & 0.001 & 0.001 & 0.001 & 0.000 & 0.002 & 0.001 & 0.001 & 0.003 & 0.001 & 0.002 & 0.000 \\
\hline $\mathrm{Mg}^{2+}$ & 0.133 & 0.163 & 0.051 & 0.109 & 0.001 & 0.012 & 0.216 & 0.168 & 0.234 & 0.240 & 0.144 \\
\hline$\Sigma M$ & 2.000 & 2.000 & 2.000 & 2.000 & 2.000 & 2.000 & 2.000 & 2.000 & 2.000 & 2.000 & 2.000 \\
\hline $\mathrm{Ca}^{2+}$ & 0.001 & 0.001 & 0.003 & 0.002 & 0.001 & 0.000 & 0.004 & 0.006 & 0.002 & 0.004 & 0.002 \\
\hline $\mathrm{Na}^{+}$ & 0.003 & 0.010 & 0.116 & 0.080 & 0.100 & 0.077 & 0.008 & 0.024 & 0.005 & 0.007 & 0.047 \\
\hline $\mathrm{K}^{+}$ & 0.964 & 0.937 & 0.749 & 0.787 & 0.755 & 0.815 & 0.954 & 0.926 & 0.895 & 0.815 & 0.844 \\
\hline$\square$ & 0.032 & 0.051 & 0.132 & 0.131 & 0.143 & 0.109 & 0.034 & 0.044 & 0.098 & 0.173 & 0.107 \\
\hline$\Sigma I$ & 0.968 & 0.949 & 0.868 & 0.869 & 0.857 & 0.891 & 0.966 & 0.956 & 0.902 & 0.827 & 0.893 \\
\hline $\mathrm{OH}^{-}$ & 2.000 & 2.000 & 2.000 & 2.000 & 2.000 & 2.000 & 2.000 & 2.000 & 2.000 & 2.000 & 2.000 \\
\hline
\end{tabular}

Classification of white micas after Tischendorf et al. (2007); we often use phengite (Phg) as an equivalent of Cel-rich Ms; sample location after Figure 2; depicted position of dated samples PI-2F, PI-4A, PI-14A; position of the other samples: PI-16 (0.5 km west of Čierny vrch Hill), $\mathrm{PI}-\mathrm{B} 3 \mathrm{a}$ (1.3 km east of PI-4F), PI-R11b (tiny meta-rhyolite body SE of PI-2F), PI-RD1 (1.5 km east of PI-22A) 
Tab. 4 cont

Representative EPMA of white micas from the Lower and Upper Cretaceous rocks

\begin{tabular}{|c|c|c|c|c|c|c|c|c|}
\hline \multirow{3}{*}{$\begin{array}{c}\text { Sample } \\
\text { Age } \\
\text { Rock } \\
\end{array}$} & \multicolumn{2}{|c|}{$\mathrm{PI}-21 \mathrm{a}$} & \multirow{2}{*}{\multicolumn{2}{|c|}{$\frac{\mathrm{Pl}-39-7}{\text { etaceous }}$}} & $\mathrm{PI}-136$ & \multicolumn{2}{|c|}{$\mathrm{PI}-88 \mathrm{a}$} & $\mathrm{Pl}-88 \mathrm{~b}$ \\
\hline & \multicolumn{2}{|c|}{ Lower Cretaceous } & & & \multicolumn{4}{|c|}{ Upper Cretaceous } \\
\hline & \multicolumn{2}{|c|}{ Slate in Flysch } & \multicolumn{3}{|c|}{ Couches Rouges Shale } & \multicolumn{3}{|c|}{ Flysch Shale } \\
\hline Mineral & Cel-Ms & Cel-Ms & Ms clast & III & Ms clast & Cel-Ms clast & III & III \\
\hline \multicolumn{9}{|c|}{ [wt.\%] } \\
\hline $\mathrm{SiO}_{2}$ & 50.52 & 50.85 & 48.03 & 51.89 & 49.01 & 51.77 & 46.31 & 43.42 \\
\hline $\mathrm{TiO}_{2}$ & 0.12 & 0.64 & 0.48 & 0.07 & 0.16 & 0.43 & 1.03 & 0.38 \\
\hline $\mathrm{Al}_{2} \mathrm{O}_{3}$ & 30.14 & 28.06 & 34.76 & 29.62 & 34.99 & 24.91 & 30.04 & 24.01 \\
\hline $\mathrm{FeO}$ & 2.98 & 3.51 & 1.05 & 3.50 & 1.31 & 2.54 & 4.95 & 8.50 \\
\hline $\mathrm{MnO}$ & 0.06 & 0.05 & 0.00 & 0.00 & 0.05 & 0.00 & 0.20 & 0.10 \\
\hline $\mathrm{MgO}$ & 2.96 & 2.61 & 0.80 & 2.35 & 1.08 & 3.41 & 2.67 & 5.65 \\
\hline $\mathrm{CaO}$ & 0.08 & 0.07 & 0.02 & 0.11 & 0.25 & 0.75 & 0.29 & 0.94 \\
\hline $\mathrm{Na}_{2} \mathrm{O}$ & 0.11 & 0.16 & 0.70 & 0.21 & 0.45 & 0.28 & 0.32 & 0.10 \\
\hline $\mathrm{K}_{2} \mathrm{O}$ & 9.07 & 9.21 & 7.82 & 7.41 & 8.73 & 9.43 & 7.23 & 4.95 \\
\hline $\mathrm{H}_{2} \mathrm{O}^{*}$ & 4.56 & 4.50 & 4.52 & 4.57 & 4.61 & 4.43 & 4.38 & 4.08 \\
\hline Total & 100.61 & 99.67 & 98.18 & 99.72 & 100.64 & 97.94 & 97.43 & 92.12 \\
\hline \multicolumn{9}{|c|}{ Based on 11 oxygen (apfu) } \\
\hline $\mathrm{Si}^{4+}$ & 3.319 & 3.385 & 3.183 & 3.403 & 3.187 & 3.504 & 3.168 & 3.190 \\
\hline${ }^{\mathrm{IV}} \mathrm{Al}^{3+}$ & 0.681 & 0.615 & 0.817 & 0.597 & 0.813 & 0.496 & 0.832 & 0.810 \\
\hline$\Sigma T$ & 4.000 & 4.000 & 4.000 & 4.000 & 4.000 & 4.000 & 4.000 & 4.000 \\
\hline $\mathrm{Mg}^{2+}$ & 0.116 & 0.076 & 0.059 & 0.118 & 0.055 & 0.001 & 0.210 & 0.437 \\
\hline 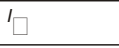 & 0.884 & 0.924 & 0.941 & 0.882 & 0.945 & 0.999 & 0.790 & 0.563 \\
\hline $\mathrm{Ti}^{4+}$ & 0.006 & 0.032 & 0.024 & 0.003 & 0.008 & 0.022 & 0.053 & 0.021 \\
\hline${ }^{\mathrm{VI}} \mathrm{Al}^{3+}$ & 1.653 & 1.587 & 1.898 & 1.693 & 1.869 & 1.491 & 1.590 & 1.269 \\
\hline $\mathrm{Fe}^{2+}$ & 0.164 & 0.195 & 0.058 & 0.192 & 0.071 & 0.144 & 0.283 & 0.522 \\
\hline $\mathrm{Mn}^{2+}$ & 0.003 & 0.003 & 0.000 & 0.000 & 0.003 & 0.000 & 0.012 & 0.006 \\
\hline $\mathrm{Mg}^{2+}$ & 0.174 & 0.183 & 0.020 & 0.112 & 0.049 & 0.343 & 0.062 & 0.182 \\
\hline$\Sigma M$ & 2.000 & 2.000 & 2.000 & 2.000 & 2.000 & 2.000 & 2.000 & 2.000 \\
\hline $\mathrm{Ca}^{2+}$ & 0.005 & 0.005 & 0.001 & 0.008 & 0.018 & 0.054 & 0.021 & 0.074 \\
\hline $\mathrm{Na}^{+}$ & 0.014 & 0.020 & 0.090 & 0.027 & 0.057 & 0.036 & 0.043 & 0.014 \\
\hline $\mathrm{K}^{+}$ & 0.760 & 0.782 & 0.661 & 0.620 & 0.725 & 0.814 & 0.631 & 0.463 \\
\hline 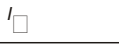 & 0.220 & 0.193 & 0.248 & 0.346 & 0.201 & 0.096 & 0.305 & 0.449 \\
\hline$\Sigma I$ & 0.780 & 0.807 & 0.752 & 0.654 & 0.799 & 0.904 & 0.695 & 0.551 \\
\hline $\mathrm{OH}^{-}$ & 2.000 & 2.000 & 2.000 & 2.000 & 2.000 & 2.000 & 2.000 & 2.000 \\
\hline
\end{tabular}

Sample location: PI-21a (the same outcrop as dated sample PI-22A SE of Čierny vrch Hill), PI-39-7 (1 km NNE of Humienec Hill, see also Fig. 6), PI-136 (0.5 km NW of Humienec Hill), PI-88a, PI-88b (1.25 km SE of Čierny vrch Hill)

We also describe 5 representative $\mathrm{Ar}-\mathrm{Ar}$-dated samples in greater detail (Fig. 12) from Putiš et al. (2009a), which are important for definition of the (D) Infratatric evolutionary stages. $\mathrm{Ar}-\mathrm{Ar}$ analyses were performed by measuring the ${ }^{40} \mathrm{Ar} * / 39 \mathrm{Ar}$ isotopic ratio of mica aggregates at the Central European Ar-Laboratory - CEAL of the Earth Science (former Geological) Institute of The Slovak Academy of Sciences in Bratislava. The analytical procedure was briefly published in Putiš et al. (2009a) and Faryad and Frank (2011). Here we provide new information on sample choice, white mica separation, elimination of irradiation recoil effects, and $\mathrm{K} / \mathrm{Ca}$ ratio calculation.

Only the following two-phase rocks were chosen for $\mathrm{Ar}-\mathrm{Ar}$ dating of fine-grained anchi-metamorphic white mica aggregates: Permian quartzitic meta-sandstones (PI-4, Pl-14) and Lower Cretaceous cherty slates (PI-22) from the Infratatric Inovec Nappe cover. In addition to quartz, these rocks contain only phengite (PI-4), muscovite (PI-14) or illite-phengite (PI-22). This avoided Ar gain from other phases such as feldspar and mica porphyroclasts. EPMA analysis revealed that white mica is a newly formed homogeneous generation, and its separation commenced with careful manual rock-crushing to avoid "over-crushing". Fine-grained fractions in $<2 \mu \mathrm{m}, 5-10$ and $20 \mu \mathrm{m}$ size were separated by sedimentation in distilled water, and then most of the under-size fraction was cut off. We employed a special arrangement of encapsulated samples to eliminate recoil effect and this captured all quantitative Ar loss during the irradiation procedure, as in Foland et al. (1992). We assumed possible combination of minority recoil effect with old lattice degassing, especially at higher temperature steps. The raw data correction includes time-dependent correction of radioactive decay and interfering masses. While all single-step results in the final age calculation are quoted with $1 \sigma$ errors, the 
Table 5

Chlorite thermometry results

\begin{tabular}{|c|c|c|c|c|c|}
\hline Sample & $\mathrm{P} 13 / 3$ & $\mathrm{P} 13 / 12$ & PI-R11b & PI-RD2b & $\mathrm{PI}-14$ \\
\hline Rock age & \multicolumn{5}{|c|}{ Permian } \\
\hline Rock & Basalt & Basalt & Rhyolite & Rhyodacite & Sandstone \\
\hline \multicolumn{6}{|c|}{ [wt.\%] } \\
\hline $\mathrm{SiO}_{2}$ & 28.24 & 28.81 & 27.29 & 27.99 & 25.63 \\
\hline $\mathrm{TiO}_{2}$ & 0.02 & 0.00 & 0.13 & 0.06 & 0.15 \\
\hline $\mathrm{Al}_{2} \mathrm{O}_{3}$ & 18.55 & 17.81 & 20.68 & 18.51 & 21.25 \\
\hline $\mathrm{FeO}$ & 23.63 & 22.53 & 27.59 & 22.70 & 28.88 \\
\hline $\mathrm{MnO}$ & 0.36 & 0.50 & 0.44 & 0.08 & 0.16 \\
\hline $\mathrm{MgO}$ & 17.80 & 19.00 & 11.63 & 17.41 & 9.33 \\
\hline $\mathrm{CaO}$ & 0.02 & 0.02 & 0.05 & 0.03 & 0.10 \\
\hline $\mathrm{Na}_{2} \mathrm{O}$ & 0.00 & 0.00 & 0.02 & 0.00 & 0.02 \\
\hline $\mathrm{K}_{2} \mathrm{O}$ & 0.00 & 0.00 & 0.26 & 0.02 & 0.30 \\
\hline $\mathrm{H}_{2} \mathrm{O}^{*}$ & 11.64 & 11.56 & 11.34 & 11.47 & 10.93 \\
\hline Total & 100.26 & 99.03 & 99.43 & 98.27 & 96.74 \\
\hline \multicolumn{6}{|c|}{ Based on 14 anions (apfu) } \\
\hline $\mathrm{Si}^{4+}$ & 2.909 & 2.989 & 2.887 & 2.928 & 2.813 \\
\hline${ }^{\mathrm{IV}} \mathrm{Al}^{3+}$ & 1.091 & 1.011 & 1.113 & 1.072 & 1.187 \\
\hline$\Sigma T$ & 4.000 & 4.000 & 4.000 & 4.000 & 4.000 \\
\hline $\mathrm{Ti}^{4+}$ & 0.002 & 0.000 & 0.010 & 0.005 & 0.012 \\
\hline${ }^{\mathrm{V}} \mathrm{Al}^{3+}$ & 1.160 & 1.167 & 1.465 & 1.210 & 1.562 \\
\hline $\mathrm{Fe}^{2+}$ & 2.035 & 1.955 & 2.440 & 1.986 & 2.651 \\
\hline $\mathrm{Mn}^{2+}$ & 0.031 & 0.044 & 0.040 & 0.007 & 0.015 \\
\hline $\mathrm{Mg}^{2+}$ & 2.733 & 2.753 & 1.833 & 2.714 & 1.527 \\
\hline $\mathrm{Ca}^{2+}$ & 0.002 & 0.002 & 0.006 & 0.003 & 0.012 \\
\hline $\mathrm{Na}^{+}$ & 0.000 & 0.000 & 0.005 & 0.000 & 0.004 \\
\hline $\mathrm{K}^{+}$ & 0.000 & 0.000 & 0.035 & 0.003 & 0.042 \\
\hline$\Sigma M$ & 5.962 & 5.920 & 5.834 & 5.928 & 5.824 \\
\hline \multirow[t]{2}{*}{$\mathrm{OH}^{-}$} & 8.000 & 8.000 & 8.000 & 8.000 & 8.000 \\
\hline & Clinochlore & Clinochlore & Chamosite & Clinochlore & Chamosite \\
\hline Mg\# & 0.43 & 0.46 & 0.30 & 0.43 & 0.24 \\
\hline$T_{1}\left[{ }^{\circ} \mathrm{C}\right]$ - Cathelineau (1988) & 289 & 263 & 296 & 283 & 321 \\
\hline$T_{2}\left[{ }^{\circ} \mathrm{C}\right]-$ Jowett $(1991)$ & 293 & 267 & 304 & 287 & 330 \\
\hline
\end{tabular}

Classification of chlorite after Zane and Weiss (1998)

Fig. 9. Metamorphosed rocks of the Inovec Nappe (A, C-H) as olistoliths, lithoclasts and scarp-breccia clasts in Upper Cretaceous flysch (B)

A - Permian meta-basalt with S1 metamorphic schistosity (D1) Chl flakes and relict porphyroclasts of flattened/stretched and sinistrally rotated amygdaloids (sample PI-3A); B - scarp breccia (D3-related) composed of Permian meta-basalt (s. PI-BS1) and marble clasts in the Upper Cretaceous red pelagic clay pseudo-matrix bottom layer with slumping deformation structures; C - reddish schistose folded Upper Jurassic meta-radiolarite (D1) of the Humienec Succession (s. PI-21); D - dark grey Lower Cretaceous folded cherty slate (D1) of the Humienec Succession (s. PI-22); E - meta-radiolarite (D1) texture from (C) with recrystallized radiolarians in S1 schistosity with illite-phengite aggregates (s. PI-21); F - cherty slate texture with newly formed III-Phg aggregates in S1 schistosity and S2 cleavage, and recrystallized Qz layers (s. PI-22); G - Permian meta-sandstone with metamorphic S1 schistosity (D1) phengite aggregates (s. PI-4); H Permian meta-basalt with metamorphic S1 schistosity (D1) Chl, $\operatorname{Tr}(\mathrm{Act}), \mathrm{Ep}, \mathrm{Ab}$, and rare Ce-Ms(Phg), Qz and Cal (s. Pl-3a) 

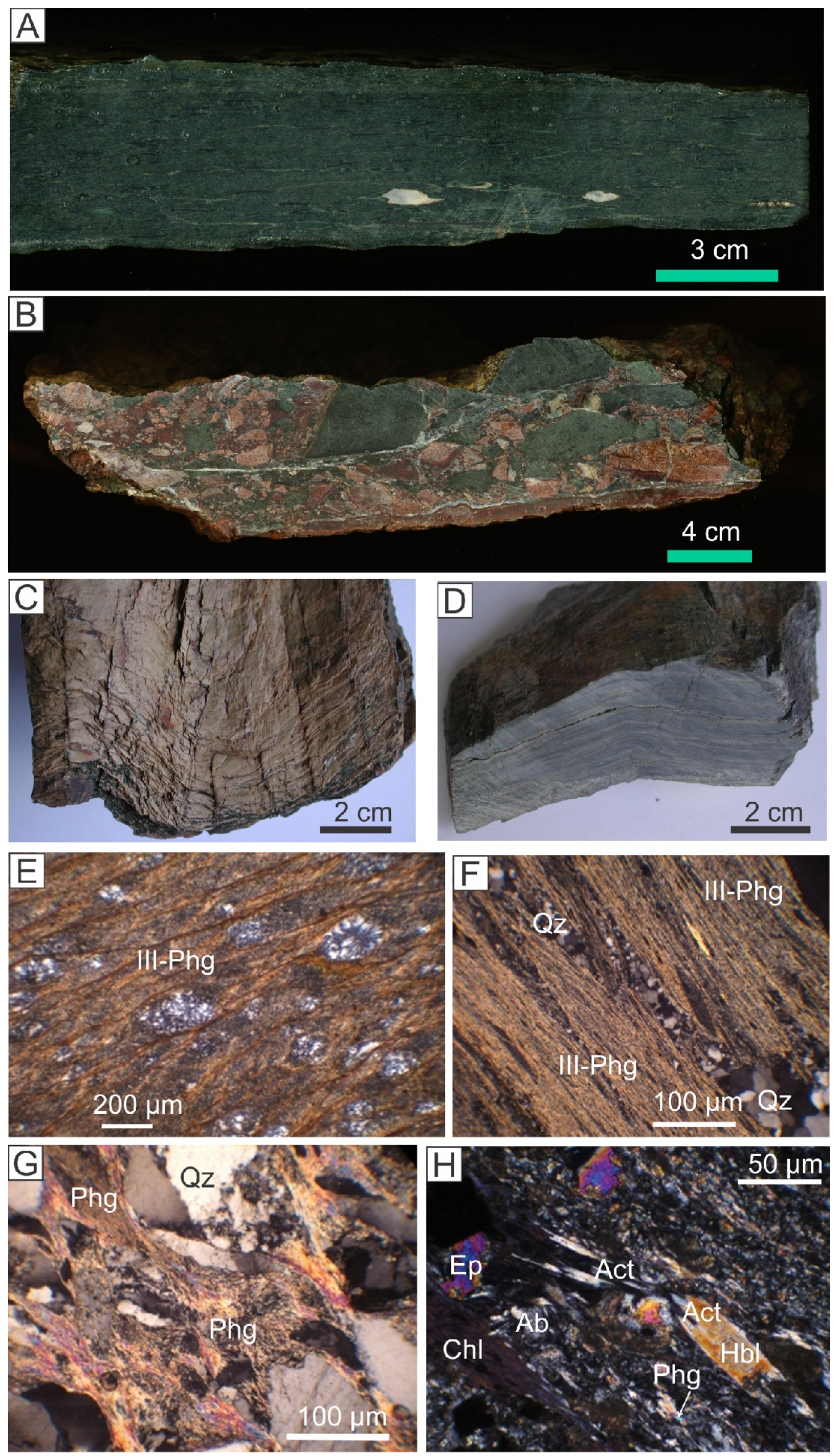

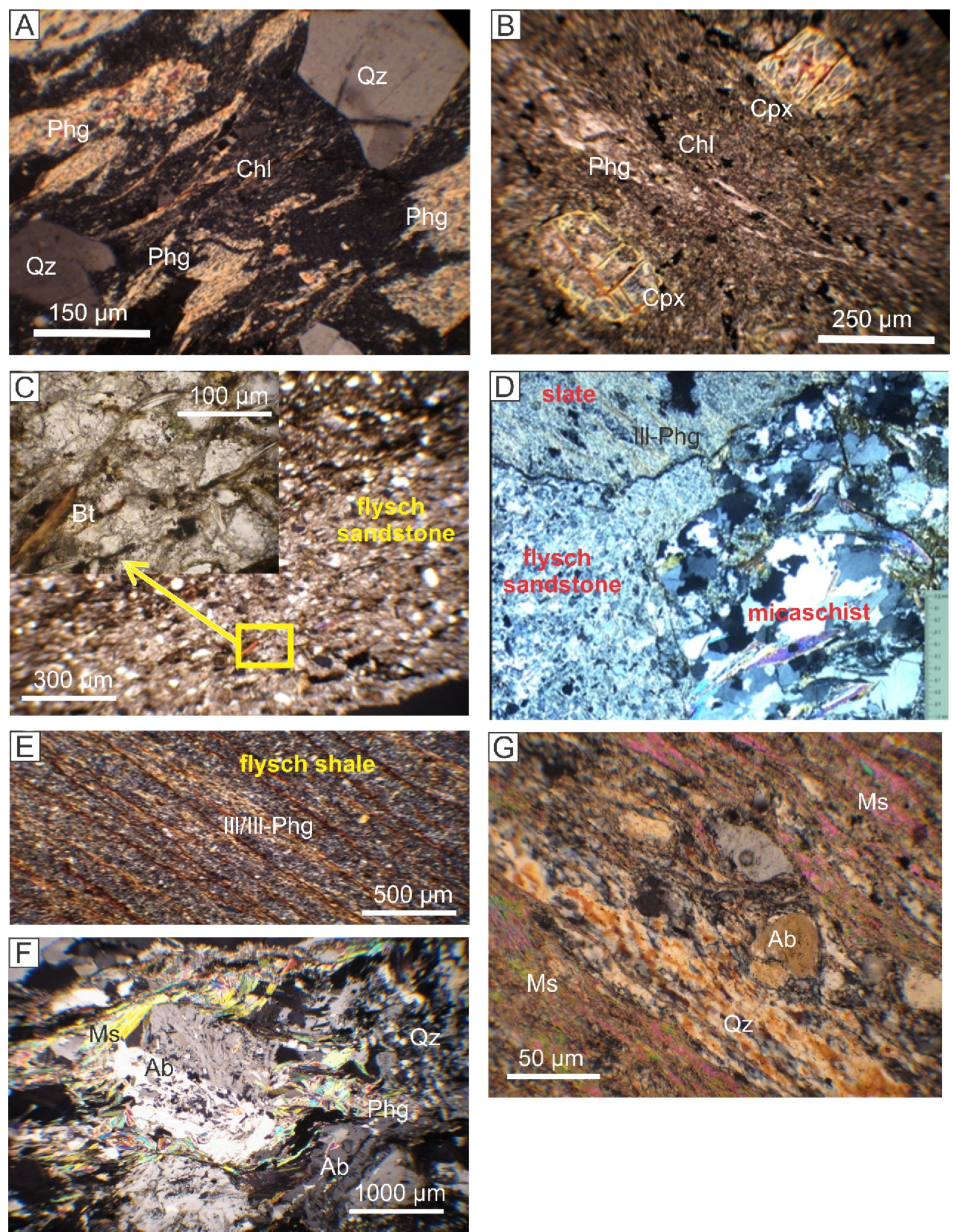

Fig. 10. Metamorphozed lithoclasts from the Inovec Nappe (A, B, D) within flysch sandy-clay pseudomatrix, the latter without a distinct metamorphic overprinting (C, D, E); Hrádok-Zlatníky thrust-fault phyllonite (F) and mylonite (G)

A-Permian meta-rhyolite clast with metamorphic S1 schistosity (D1) Cel-Ms(Phg) aggregates (sample PI-RS1) as a part of Permian olistolith in Upper Cretaceous Belice flysch; B - Permian meta-basalt clast with metamorphic S1 schistosity (D1) Chl aggregates (s. PI-BS1) as a part of Permian olistolith in Upper Cretaceous Belice flysch; C - Upper Cretaceous flysch sandstone (s. PI-88b) well-preserving clastogeneous texture of Ms, Bt, Qz and Cal (a detail in the upper left corner); D - Upper Cretaceous flysch sandy pseudomatrix of scarp breccia with clasts of Lower Cretaceous cherty slates (top, with metamorphic D1 III-Phg) and micaschists; E - Upper Cretaceous flysch shale with diagenetic to lowest anchi-metamorphic III to III-Phg (s. PI-88a); F - phyllonitized micaschist with newly formed albite porphyroblasts and fine-flaked $\mathrm{Cel}-\mathrm{Ms}(\mathrm{Phg})$ aggregates replacing $\mathrm{Ms}$; G - granite blastomylonite of the Tatric Panská Javorina Nappe hanging wall with newly formed fine-flaked Ms aggregates alternating with dynamically recrystallized Qz layers (s. PI-17HZ) 


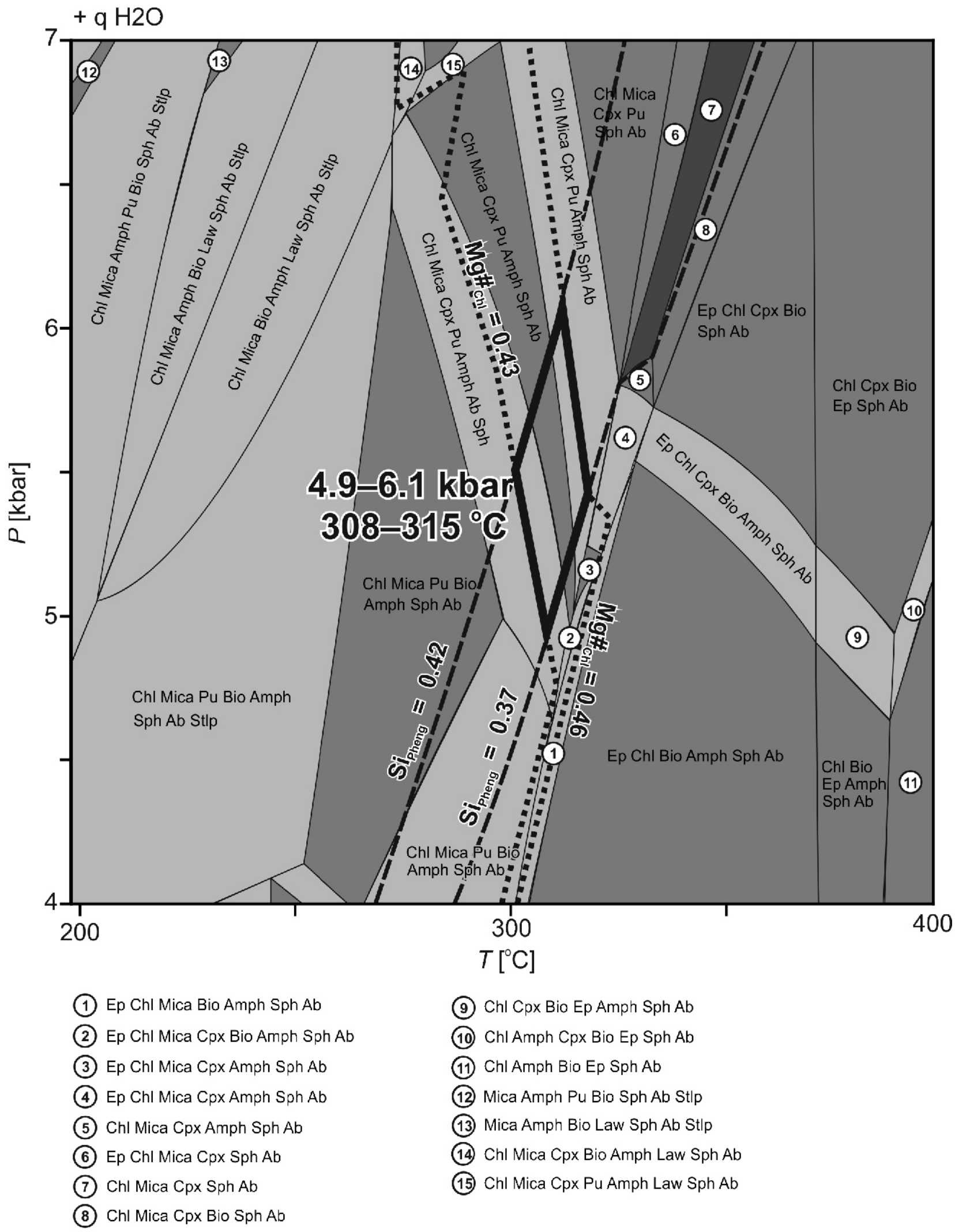

Fig. 11. The pseudosection P-T calculation diagram by Perple_X 


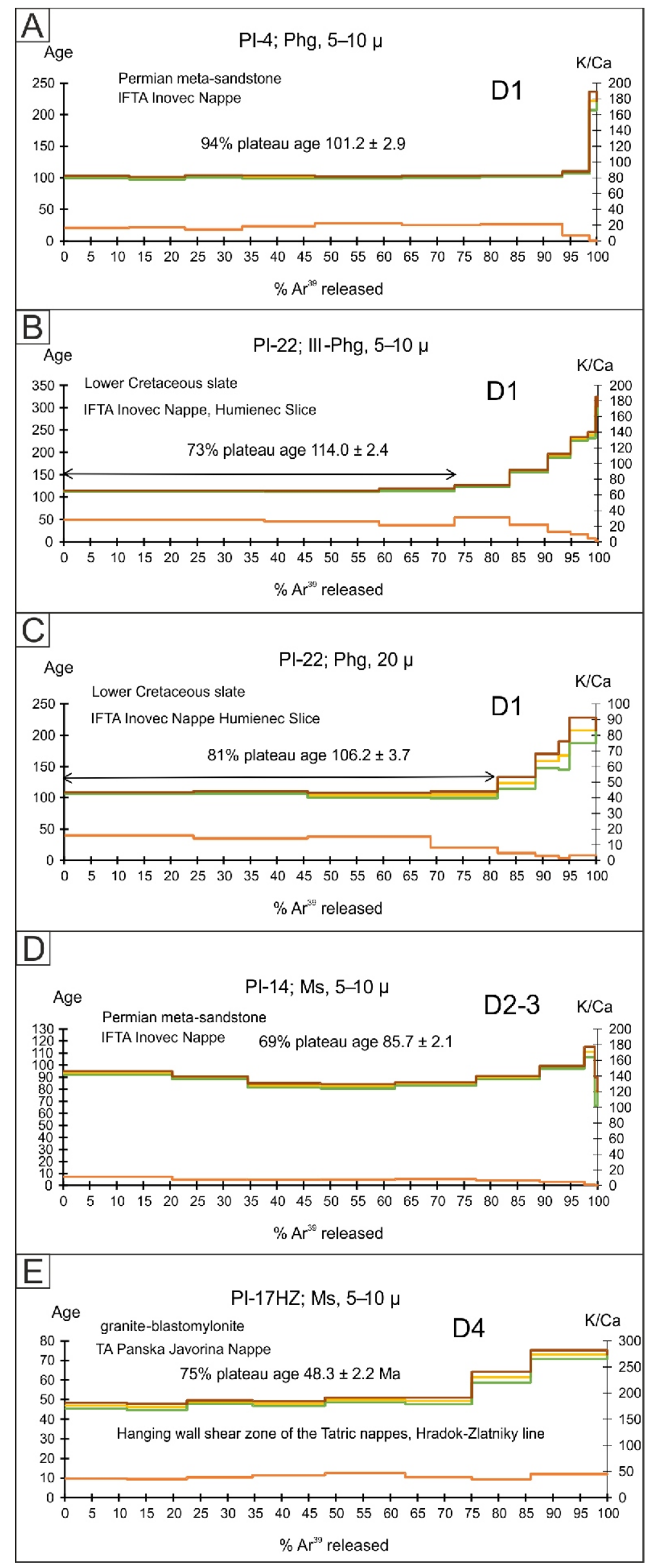

Fig. 12. Representative ${ }^{40} \mathrm{Ar}-{ }^{39} \mathrm{Ar}$ age diagrams for D-stages: A-C - D1 stage (115-9 5Ma); D - D2 (95-85 Ma) to D3 (85-65 Ma) stages; E - D4 stage (65-45 Ma), taken from Putiš et al. (2009a) 
calcuated plateau ages include an additional $\pm 0.4 \%$ error on the $\mathrm{J}$ value to compensate for systematic variations in standard sample experiments. Correction factors were determined from optical-grade $\mathrm{CaF}_{2}$ and are: ${ }^{36} \mathrm{Ar} /{ }^{37} \mathrm{Ar}(\mathrm{Ca})=0.00022,{ }^{39} \mathrm{Ar} /{ }^{37} \mathrm{Ar}$ $(\mathrm{Ca})=0.00039,{ }^{40} \mathrm{Ar} /{ }^{39} \mathrm{Ar}(\mathrm{K})=0.0254$. The $\mathrm{K} / \mathrm{Ca}$ ratio in the diagrams is based on a conversion factor of 0.247 . There is very good correlation in most spectra, with older ages for lower $\mathrm{K} / \mathrm{Ca}$ ratios (Ar deficiency relative to $\mathrm{K}$ related to $\mathrm{Ar}$ loss).

All samples were tested for $\mathrm{Cl}$ before irradiation and each $\mathrm{Ar}$ gas release is plotted as a bar. The length of the bar establishes its volume as a fraction of the total ${ }^{39} \mathrm{Ar}$ released from the sample, and the value forms the corrected ${ }^{40} \mathrm{Ar} /{ }^{39} \mathrm{Ar}$ isotopic ratio.

\section{RESULTS AND INTERPRETATION}

\section{ZFT DATA}

The ZFT analysis results are listed in Tables 1 and 2 and displayed in Figure 8. Of the six samples, only PI-6 (Campanian sandstone from flysch) and PI-9 (Variscan granite) passed the chi-square test at $>95 \%$ confidence and therefore comprise one age population with Early Cretaceous central ages of $142.5 \pm 7.5 \mathrm{Ma}(\mathrm{PI}-6)$ and $133.2 \pm 6.9 \mathrm{Ma}(\mathrm{PI}-9)$. The interpretation of our ZFT ages, however, is not unambiguous because of the lack of track length data which provides useful indication of the samples thermal history. These Early Cretaceous ages are temporally consistent with an extension event and can therefore be interpreted as cooling ages related to an exhumation. Alternatively, the Early Cretaceous ages may be an apparent result of a partial reset by Late Cretaceous thermal overprinting.

The remaining samples had complex single-grain age spectra with high dispersion, and they passed the chi-square test at low $<35 \%$ confidence level or failed it; thus, suggesting a complex thermal history and/or the presence of multiple age populations that cannot be identified within a central age. All samples display weak negative linear correlation between single-grain FT ages and $U$ content (Fig. 8), suggesting that grains with higher accumulated radiation damage may have recorded cooling below lower closure temperatures than grains with less damage. Component analysis of these $\mathrm{PI}-2,4,5$ and 12 samples revealed several populations of Jurassic ( 176 and $\sim 148 \mathrm{Ma})$, late Early Cretaceous ( 109 Ma), Late Cretaceous ( $\sim 83$ and $\sim 75 \mathrm{Ma})$, late Paleocene ( $\sim 57 \mathrm{Ma})$ and Eocene ( 47 and $\sim 37 \mathrm{Ma}$ ) ages (Table 2 and Fig. 8). Again, in the absence of track length data we can only speculate whether these age populations are related to a distinct thermal event or are merely apparent cooling ages from a different degree of partial rejuvenation of the FT thermochronometer in zircons with a different amount of radiation damage. However, since the identified age populations tend to coincide with some known geological events and are in some cases similar to the ages determined by $\mathrm{Ar}-\mathrm{Ar}$ and $\mathrm{K}-\mathrm{Ar}$ methods, we tentatively interpret these ages as cooling ages.

Regardless of the precise meaning of the ZFT ages, the presence of the Cretaceous and Paleocene-Eocene populations in these four samples clearly documents the Alpine overprint in the Selec Block. This conclusion agrees with the geological observations, metamorphic assemblages and ${ }^{40} \mathrm{Ar}-{ }^{39} \mathrm{Ar}$ data, and contradicts the lack of Alpine overprint in the Infratatric Selec Block, as proposed by Králiková et al. (2016).

TECTONO-METAMORPHIC OVERPRINTING GRADE OF THE INFRATATRICUM

The EPMA results are related to newly formed (Alpine) metamorphic overprinting textures of the basement mica- schist-gneisses changed to albite schists, and infolded Upper Paleozoic and Mesozoic cover rocks (Tables 3, 4 and Figs. 9, 10). Similar metamorphic textures were found from clasts to olistoliths of the basement and Upper Carboniferous to Lower Cretaceous cover-rocks in Upper Cretaceous flysch. The distinctness of macroscopic metamorphic features is variable because samples are from an anchi-metamorphic terrain, and they vary from slightly to strongly sheared or schistose rocks; with the latter containing increased metamorphic mineral content. Constraining metamorphic conditions is most important in interpreting the ${ }^{40} \mathrm{Ar}-{ }^{39} \mathrm{Ar}$ and ZFT ages of this area; the following three metamorphic overprinting grades are distinguished:

(1) The highest anchi-metamorphic (VLT-LT/MP) overprint was determined from Permian basalts of the Inovec Nappe, which have schistose structures defined by Ep-Czo, Chl, low-Al $\operatorname{Tr}$ ( Act; approximately 1 wt. $\% \mathrm{Al}_{2} \mathrm{O}_{3}$ ), Ab, carbonate (Cal), Ttn (leucoxene), F-Ap, sagenite, Mag and rare Cel-rich Ms ("Phg"), minor contents of Pmp (Fig. 9 and Tables 3,4) and relics of magmatic $\mathrm{Hbl}$ and $\mathrm{Cpx}$ which are almost completely replaced by Chl, Act, Ep-Czo and Cal aggregates. Fine-grained aggregates of the metamorphic S1 schistosity are often crosscut by coarser-grained metamorphic veinlets composed of the above-mentioned phases. Further indication of the very low-temperature greenschist facies is supplied by the cataclasis, clast resolution and partial recrystallization observed in detrital Permian coarse-clastic sediments such as sandstones to conglomerates and rhyolite pyroclastics with fibrous aggregates of Ser-Phg, Chl, Ab and Qz grown into metamorphic schistosity. The Permian fine-grained quartzitic sandstones are rich in compositionally homogeneous "phengitic" mica aggregates in the D1 (anchi-)metamorphic stage S1 schistosity (Fig. 9 and Table 4). This white mica was suitable for ${ }^{40} \mathrm{Ar}-{ }^{39} \mathrm{Ar}$ dating (s. Pl-4; Putiš et al., 2009a and Discussion herein).

The estimated metamorphic conditions, based solely on a typical lower greenschist-facies mineral assemblage, are approximately $250-300^{\circ} \mathrm{C}$. The estimated burial depth would be approximately $12-15 \mathrm{~km}$ if a $20^{\circ} \mathrm{C} / \mathrm{km}$ gradient in the late Early Cretaceous D1 stage is assumed. The estimated pressure would be $\sim 5-7$ kbar for "phengitic" white mica with Si 3.3-3.5 p.f.u. from the Permian meta-rhyolite (Fig. 10A and Table 4) containing relic albitized $\mathrm{Kfs}$ and chloritized $\mathrm{Bt}$ porphyroclasts. The phengite barometer after Massonne and Schreyer (1987) was employed in this temperature interval, with $293^{\circ} \mathrm{C}$ average temperature obtained from chlorite thermometry (Table 5).

We applied Perple_X pseudo-section modelling to determine the precise D1 stage P-T conditions and obtained $308-315^{\circ} \mathrm{C}$ at $4.9-6.1 \mathrm{kbar}$ at approximately $15-18 \mathrm{~km}$ burial and $17-20^{\circ} \mathrm{C} / \mathrm{km}$ gradient (Fig. 11). These estimates are from the Permian meta-basalt of the Inovec Nappe (s. PI-B3a; Fig. 9A, H) and they are also valid for the meta-basalt fragments present in olistoliths in the Upper Cretaceous flysch sediments (s. PI-BS1; Fig. 10B).

(2) The medium anchi-metamorphic conditions (D1 stage) of $200-250^{\circ} \mathrm{C}$ of the Inovec Nappe were estimated from Lower Cretaceous slates (s. PI-21a; Fig. 9D, F and Table 4) of the reconstructed Humienec Succession. They occur as clast-to$\mathrm{km}$-size olistolith fragments in the Upper Santonian to Maastrichtian flysch of this succession. The Lower Cretaceous slates are originally deep-water cherty sediments associated with radiolarites (Fig. 9C, E). The newly formed III-Phg metamorphic aggregate has mostly 3-25 $\mu \mathrm{m}$ stable grain-size (fig. 3A BSE image in Sulák et al., 2009) and homogeneous composition. This was therefore used for ${ }^{40} \mathrm{Ar}-{ }^{39} \mathrm{Ar}$ dating of the D1 stage (Putiš et al., 2009a; see Discussion). The III-Phg aggregates define penetrative metamorphic S1 schistosity and S2 
axial-plane slaty cleavage of syn-metamorphic D1 stage isoclinal intra-S1 folds. The presence of III-Phg (3.3-3.4 Si p.f.u.) with $0.7-0.8$ apfu $\mathrm{K}+\mathrm{Na}$ values and $8-9$ wt. $\% \mathrm{~K}_{2} \mathrm{O}$ is consistent with medium anchi-metamorphic conditions (Table 4).

(3) The lowest anchi-metamorphic conditions were achieved in the frontal part of the Infratatric Inovec Nappe (D4 stage). This is the Humienec tectonic Slice which contains Upper Cretaceous sediments (Fig. 2). These wedge-top sediments were relatively shallowly infolded into the Inovec Nappe-type basement. Here, Couches Rouges-type shales (s. PI-39-7; Table 4) and flysch sediments (s. PI-136, PI-88a, b; Fig. 10C-E and Table 4) contain the newly formed white micas (3.1-3.2 Si p.f.u.) of III-Phg composition with much lower alkalis (0.5-0.7 p.f.u.) and $\mathrm{K}_{2} \mathrm{O}$ (5-7 wt.\%) contents than in the Inovec Nappe-type basement rocks, including the Lower Cretaceous slates. Table 4 documents these low values typical of advanced diagenesis and/or lowest-temperature anchi-metamorphism at approximately $150-200^{\circ} \mathrm{C}$ (Hunziker et al., 1986; Korikovsky and Putiš, 1999).

The signatures of very low- $T$ tectono-thermal overprinting are also recognized in the Tatricum hanging wall granite blastomylonites by newly formed aggregates of dynamically recrystallized Qz, Ab, Chl and Ser-Ms (s. PI-17HZ; Fig. 10G). Newly formed homogeneous Ser-Ms (Putiš et al., 2008; Sulák et al., 2009) was suitable for ${ }^{40} \mathrm{Ar}-{ }^{39} \mathrm{Ar}$ dating of blastomylonitization (Putiš et al., 2009a and Discussion herein).

\section{DISCUSSION - CONSTRAINING THE INFRATATRIC EVOLUTIONARY STAGES}

\section{D1 STAGE RELATIONSHIP OF THE INFRATATRIC INOVEC AND THE MANÍN-KLAPE NAPPES}

The Middle Jurassic to Lower Cretaceous cover of the Humienec Succession, and the underlying Upper Carboniferous to Lower Jurassic cover-rocks of a micaschist-gneiss basement were buried within an Albian-Early Cenomanian accretionary wedge (D1 stage, $\sim 115-95 \mathrm{Ma}$ ). This wedge may have been initiated by collision of the North-Veporic-Tatric Belt with the southern Neotethyan South-Veporic-Gemeric-Meliatic wedge following the closure of the intra-continental Fatric Basin which had separated these domains until the Albian-Early Cenomanian (Putiš et al., 2009a, 2017).

The D1 evolutionary stage is constrained by the "phengitic" white mica plateau age of $101 \pm 3$ Ma obtained from the S1 metamorphic schistosity planes of a Permian meta-sandstone (s. PI-4; Figs. 9G and 12A), associated with meta-rhyolites (Fig. 10A) and meta-basalts (Figs. 9A, B, H and 10B) of the Infratatric Inovec Nappe (Putiš et al., 2009a). The Lower Cretaceous slates (s. PI-22), as metamorphosed olistolith fragments in the Upper Cretaceous Horné Belice flysch, yielded even older Albian ${ }^{40} \mathrm{Ar}-{ }^{39} \mathrm{Ar}$ plateau ages of $\sim 114$ and $106 \mathrm{Ma}$ (Putiš et al., 2009a). Both 5-10 and $20 \mu \mathrm{m}$ subfractions constrain the D1 age (115-95 Ma; Fig. 12B, C). The finer-grained fraction provides approximately $8 \mathrm{Ma}$ increased age; most likely due to recoil effect (Sperner et al., 2010; Halama et al., 2014; Scharf et al., 2016). Although this effect was mostly eliminated during irradiation, it is typically apparent in very fine-grained mica aggregates. The ages are interpreted as close to the peak D1 stage tectono-metamorphic event because the formation temperature of the white mica aggregates did not reach the inferred ${ }^{40} \mathrm{Ar}-{ }^{39} \mathrm{Ar}$ closure temperature $\left(T_{c}\right)$ of $\sim 400-450^{\circ} \mathrm{C}$ (Dallmeyer et al., 1996; Villa, 2004; Halama et al., 2014; Villa et al., 2014).

The studied sheared anchi-metamorphic rocks provide further evidence that temperature is not the sole parameter con- trolling the isotopic-ratio changes (Villa et al., 2014 and references therein). The competing processes of fluid-induced and/or deformation-induced recrystallization were more rapid than the thermally activated diffusion at much lower temperatures in the "phengite" and muscovite. Apart from these often discrete reactivation (tectono-metamorphic) zones, much older ages prevail; e.g. $\sim 325 \mathrm{Ma}{ }^{40} \mathrm{Ar}-{ }^{39} \mathrm{Ar} \mathrm{Ms}$ age from a coarse-grained micaschist at the same locality as sample PI-12 was reported by Dallmeyer et al. (1996). Similarly, Král' et al. (2013) found pre-Alpine ${ }^{40} \mathrm{Ar}-{ }^{39} \mathrm{Ar}$ (cooling?) ages of 340-305 Ma from a coarse-grained muscovite. A similar case could be Permian ZFT ages ( 256 and $255 \mathrm{Ma}$; Králiková et al., 2016) or Middle Jurassic ( 158 Ma from s. PI-12; Table 1) and Early Cretaceous ( 143 and 133 Ma from s. PI-6 and 9, respectively; Table 1) ZFT ages. Incomplete resetting of the pre-Alpine Ar-Ar ages, and even some from ZFT, is typical here and this also produced different pseudo-plateau $\mathrm{Ar}-\mathrm{Ar}$ ages between 300 and $130 \mathrm{Ma}$ (see Putiš et al., 2009a).

There are stratigraphic and palaeontological controls on the precision of ${ }^{40} \mathrm{Ar}-{ }^{39} \mathrm{Ar}$ ages obtained by us. For example, the Lower Cretaceous slates (s. PI-22) still contain recognizable remnants of Hedbergella foraminifera typical of the Cretaceous Hauterivian to Maastrichtian, and therefore the oldest age limit from the inferred recoil effect is approximately 130-120 Ma ( a cherty shale formation age). Meanwhile, the youngest age limit is provided by the post-D1 Couches Rouges-type marls from the Turonian, at $\sim 95-90 \mathrm{Ma}$. The D1 ages from samples $\mathrm{PI}-22$ ( 114 and $106 \mathrm{Ma})$ and PI-4 ( 101 Ma) exactly fall within this limiting age-interval of $\sim 120 / 115-95 / 90 \mathrm{Ma}$, and there can be only very limited influence of inferred Ar excess on the obtained plateau ages.

Our age data on the Albian-Early Cenomanian syn-metamorphic wedge D1 stage formation at approximately 115-95 Ma contradicts reconstruction of the Belice Succession in the northern (Infratatric) part of the Považský Inovec Mts., being continuous from the Lower to the Upper Cretaceous (Plašienka et al., 1994; Plašienka, 2018). We report this succession was interrupted due to the D1 stage tectono-metamorphic event; and this was suggested in Putiš et al. (2016) or Putiš and Tomek (2016). Similarly, the Váhic signatures of basalt olistoliths in flysch, inferred by Plašienka et al. (1994) or Plašienka (2018), have not been confirmed. Only the Permian dominant calc-alkaline basalt and andesito-basalt olistoliths from a destructured continental margin associated with Permian siliciclastics occur in the Upper Cretaceous flysch (Putiš et al., 2006, 2008, 2016).

The "pre-Gosauian" D1 event may have initiated formation of the thrust of the basement-cover Infratatric Inovec and the Manín-Klape rootless nappes onto a Pieninic-Váhic ( Penninic) Ocean continental margin (Figs. 13 and 14). The subduction of inferred Penninic oceanic crust started during the Turonian. This is indicated by the occurrence of "exotic" pelagic Triassic limestone pebbles in conglomerates of uppermost(?) Turonian flysch sediments in the Polish Branisko Zone of the PKB ( Kysuca to Czorsztyn units' area) immediately north of the closing Pieninic-Váhic Ocean (Birkenmajer et al., 1990).

The D1 event is likely registered in the Manín Unit Jurassic-Cretaceous sedimentary succession at approximately 5-10 Ma hiatus in the Albian, and a change of the Barremian-Aptian Urgonian shallow-water limestones to Cenomanian-Lower Turonian hemipelagic to pelagic sandy calcareous claystones and flysch with "exotic" conglomerate pebbles (Mišík and Sýkora, 1981; Marschalko and Rakús, 1997; Rakús and Marschalko, 1997). In addition, the Barremian-Aptian Urgonian shallow-water limestone pebbles in the Klape flysch, closely structurally bound to the Manín Nappe, contain Cr-spinel and 


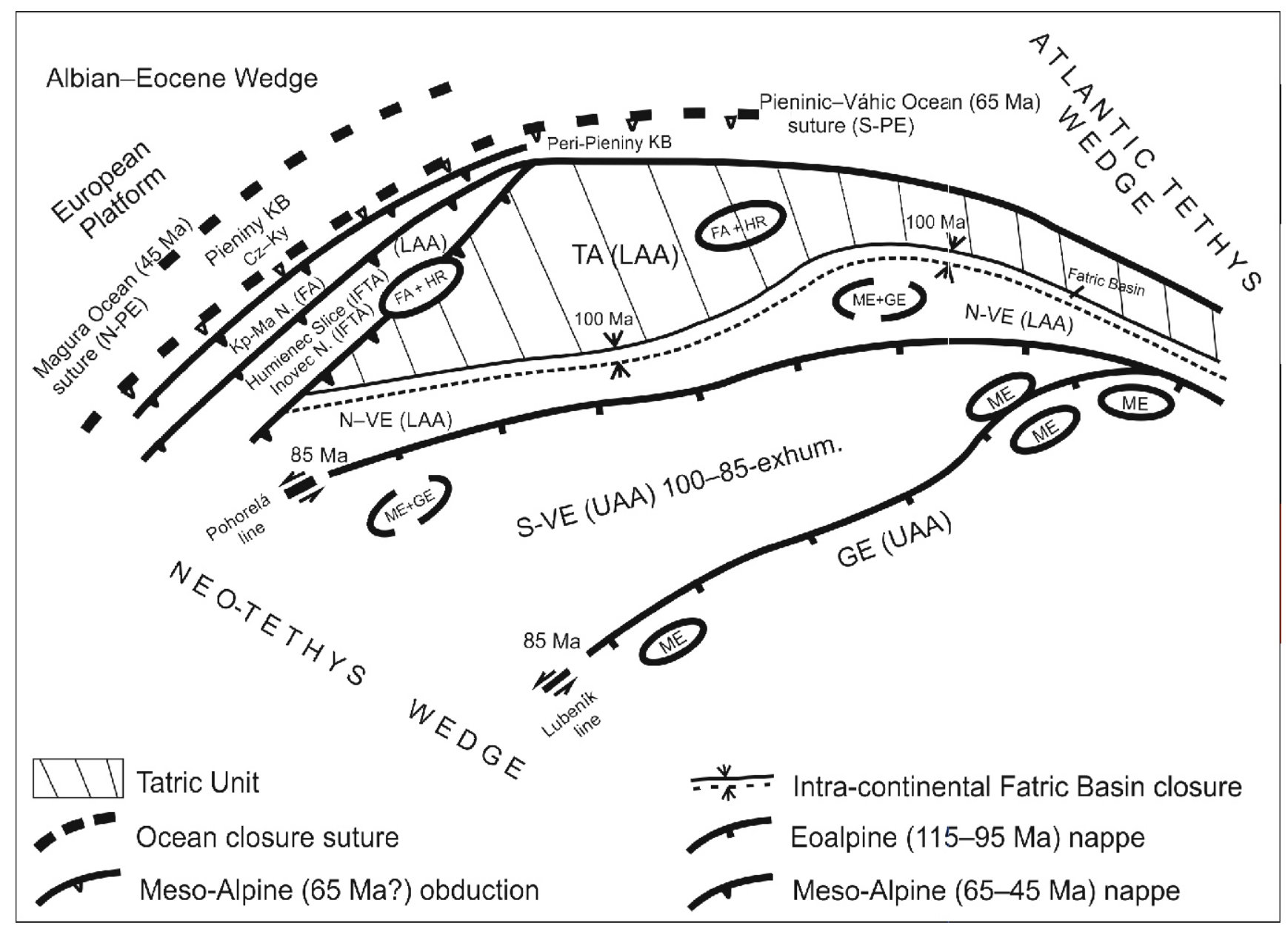

Fig. 13. Tectonic sketch of the Infratatric units

$\mathrm{Cz}$ - Czorsztyn Unit, FA - Fatric, GE - Gemeric, HR - Hronic, IFTA - Infratatric, Inovec-N - Inovec Nappe, KB - Klippen Belt, Kp-Ma Klape-Manín Nappe, Ky - Kysuca Unit, LAA - Lower Austroalpine, ME - Meliatic Bôrka Nappe, N-PE - North-Penninic, N-VE North-Veporic, S-VE - South-Veporic, S-PE - South-Penninic, TA - Tatric, UAA - Upper Austroalpine

glaucophane clasts determined by Mišík (1979) and Mišík and Sýkora (1981). However, there is no evidence about the D1 stage incorporation of the composite Manín-Klape Nappe into the Infratatric accretionary wedge or the presence of the Infratatric rock-material in the Klape flysch. Therefore, we suppose the Fatric origin of the Manín-Klape Nappe (e.g., Mahel', 1986; Plašienka, 1995, 2018) and southern sources for the Klape flysch (Plašienka, 1995, 2018; Kissová et al., 2005). Such a model is consistent with the stratigraphic extent of the "exotic" pebble-bearing conglomerate horizons in the Klape (Late Albian to Early Cenomanian) and Manín (Cenomanian to Early Turonian) flysch deposits (Marschalko and Rakús, 1997; Rakús and Marschalko, 1997) heralding the closure of the Fatric Basin. The Fatric nappes overlie the Turonian cover sediments in the Tatricum of the High Tatra, Vel'ká Fatra and the Považský Inovec Mts. Bojná Block (Pelech et al., 2017a and references therein). This record enables the Manín-Klape Nappe to reach the PPKB in the Late Turonian.

Strong compressional forces in the syn-collisional Albian-Early Cenomanian accretionary wedge were multiplied by pull forces due to underthrusting of the thermo-mechanically weaken crustal sheets. They may have caused expulsion of the Fatric wedge-top fragments over the wedging North-Veporic-Tatric-Infratatric basement towards the South-Penninic continental margin from $\sim 95$ to $90 \mathrm{Ma}$ at approximately
200-300 km distance (4-6 cm per year), following the almost contemporaneous closure of the intra-continental Fatric and Tatric-Infratatric basins (Figs. 13 and 14). These processes must have occurred before both the Late Turonian to Maastrichtian/Paleocene extension from $\sim 90$ to $85 \mathrm{Ma}$ and the formation of the new Horné Belice-type Infratatric foreland basin system above the inferred Pieninic-Váhic subduction zone (Fig. 14; Putiš et al., 2008).

Mišík and Sýkora (1981), Marschalko and Rakús (1997) and Rakús and Marschalko (1997) consider the derivation of Cr-spinel and glaucophane clasts present in the Barremian-Aptian Urgonian limestone pebbles of the Klape flysch from a Meliatic-type source which was located in the Tatric foreland. There are, however, palaeogeographic-facial (Gawlick and Missoni, 2015) as well as faunal and palaeotectonic (Csontos and Vörös, 2004) contrary arguments to a Triassic Ocean north of the IWC. Anyhow, supposing a Neotethyan, Meliatic-type source for Cr-spinel and glaucophane clasts at approximately 130-115 Ma, the questions have arisen: (1) which unit might have contained Urgonian limestones as a source for the Late Albian-Early Cenomanian Klape flysch conglomerates?, and, (2) which unit in the unlithified Barremian-Aptian limestones could receive the inferred Meliatic HP ( 160-150 Ma; Dallmeyer et al., 1996) mineral detritus and then become a source of the Klape flysch conglomerates? 
$\mathrm{N}$

S
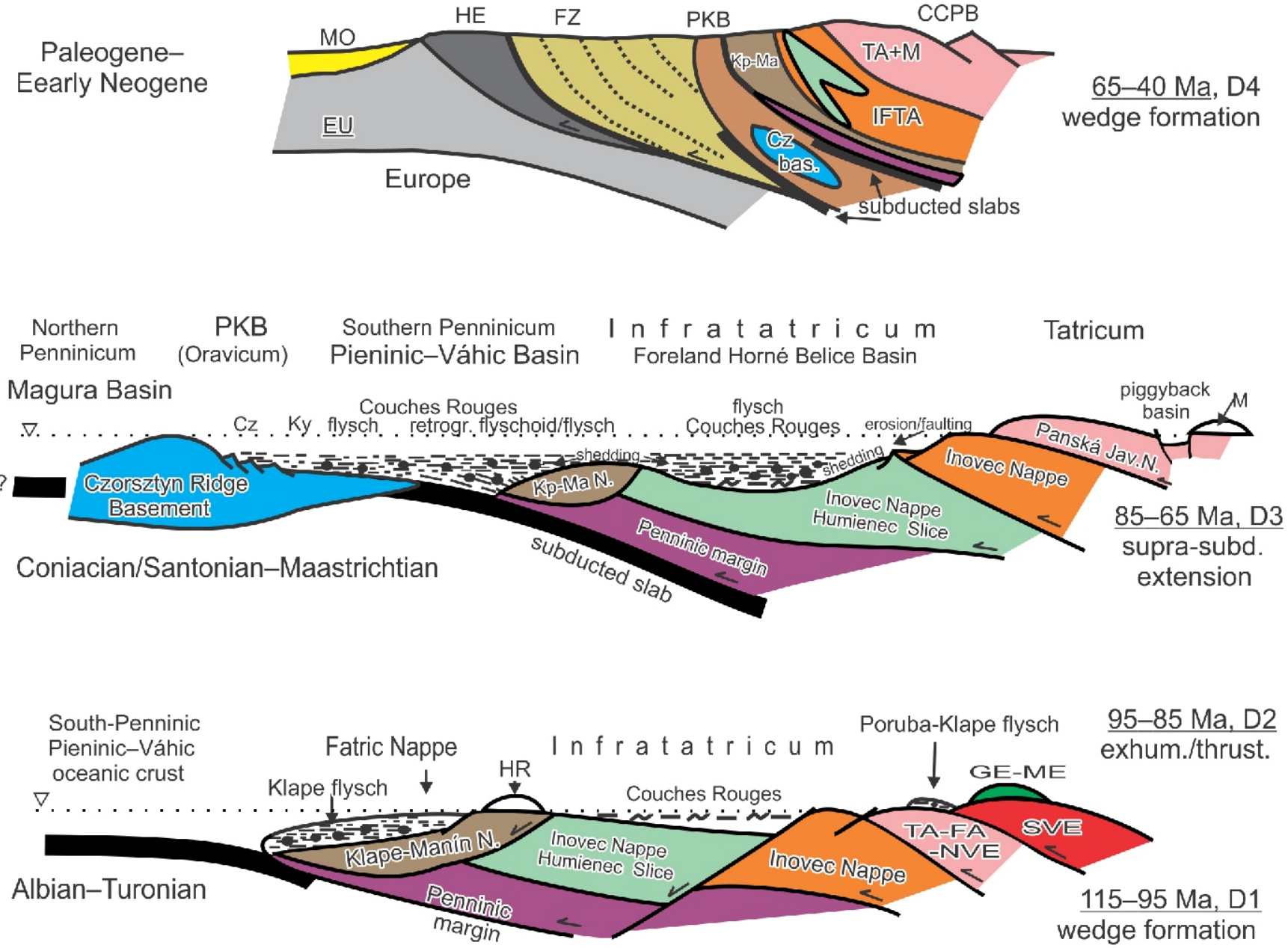

Infratatric (Humienec) Basin

IFTA-TA Swell Humienec Succession
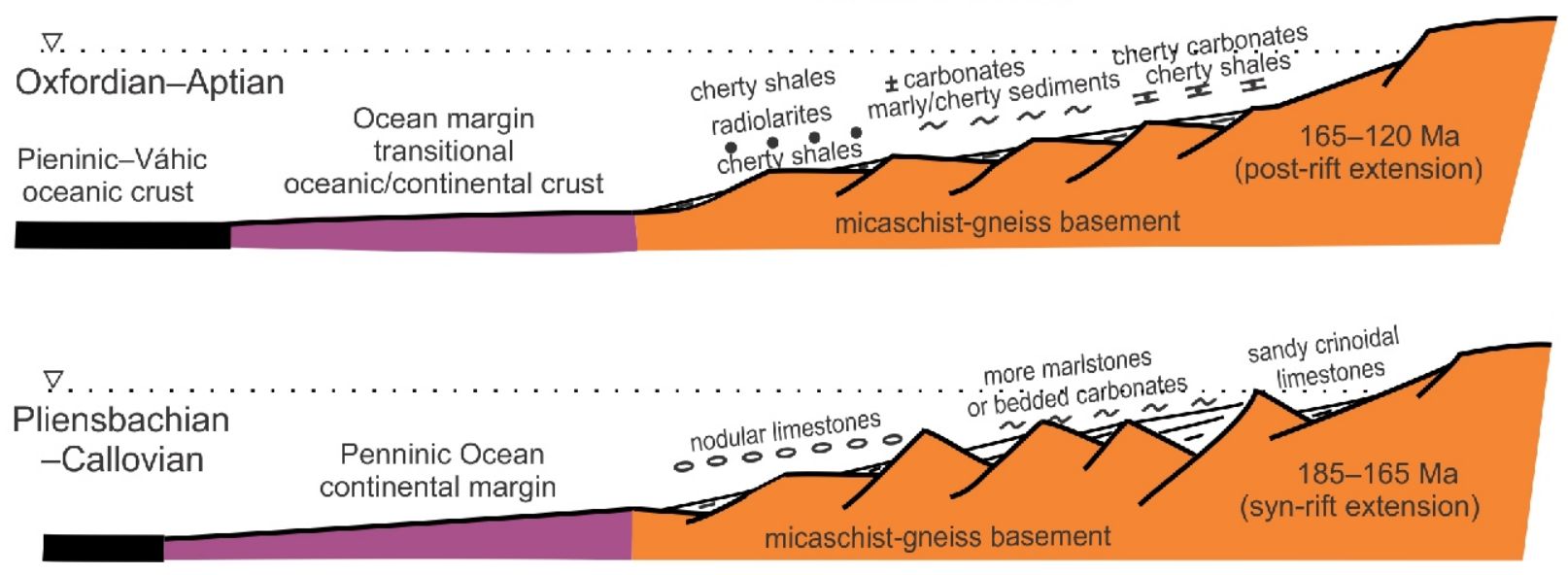

Fig. 14. Evolutionary model of the Infratatricum (modified schemes of Putiš et al., 2006, 2008)

CCPB - Central Carpathian Paleogene Basin; Cz - Czorsztyn Unit; Cz bas. - Czorsztyn Ridge basement; EU - European Plate; FA Fatricum; FZ - Outer Fysch Zone; GE - Gemeric; HE - Helvetic Zone; HR - Hronicum; IFTA - Infratatricum; Kp - Klape Unit; Ky - Kysuca Unit, Penninic margin - inferred adjacent continental zone to oceanic crust; M - Mesozoic Fatric and Hronic nappes; Ma - Manín Uint; ME Meliatic; MO - Neogene molasse; NVE - North-Veporic; PKB - Pieniny Klippen Belt; SVE - South-Veporic; TA - Tatricum, TA+M - Tatricum and Mesozoic Fatric and Hronic nappes 
Urgonian limestones of the inferred Fatric Manín Nappe and its equivalent Vysoká Succession of the Križna Nappe do not contain glaucophane and Cr-spinel clasts (Mišík and Sýkora, 1981; Rakús and Marschalko, 1997), and this could well imply that the Meliatic-type source was separated from these most likely northern margin units of the Fatric Basin by the axial deep-water zone at $130-115 \mathrm{Ma}$. However, the Fatric origin of the Klape flysch is supported by the determination of the blueschist pebbles in the Krížna Nappe Albian flysch conglomerates near Jasenov village in Eastern Slovakia close to the PKB (Ivan and Sýkora, 1993; Fig. 1).

The formation and northward thrusting of the Meliatic nappes at 140-135 Ma (Putiš et al., 2014, 2015, 2017) was initiated by underthrusting to subduction of the decoupled South-Veporic and Gemeric crustal sheets beneath the Meliatic Late Jurassic-Early Cretaceous accretionary wedge; and this is constrained by perovskite, zircon and monazite dating (Putiš et al., 2014, 2015, 2017; Vozárová et al., 2014; Hurai et al., 2015). The exhumed frontal parts of the Meliatic nappes were almost at the same structural level as the Hronic nappes forming above the South-Veporic-Gemeric basement sheets which were being sutured at that time. Urgonian limestones are unknown from the Meliatic units and the Gosau-type Senonian sediments; although these latter sediments are rich in the Meliatic-type carbonatic and ophiolitic materials near Dobšiná (Hovorka et al., 1990). However, rich fine-grained chromian spinel detritus occurs in the Hauterivian siliciclastic turbidites of the Hronicum at 133-129 Ma (Jablonský et al., 2001). These trace the Meliatic nappes overlying the Gemeric basement, and they are consistent with the South-Gemeric monazite ages of 140-135 Ma (Vozárová et al., 2014; Hurai et al., 2015). Continuing continental subduction of the decoupled South-Veporic crustal sheets below the Gemeric-Meliatic nappes is consistent with the oldest South-Veporic monazite ages at $\sim 130 \mathrm{Ma}$ (Putiš et al., 2017) and the oldest white mica ${ }^{40} \mathrm{Ar}-{ }^{39} \mathrm{Ar}$ ages of $\sim 125 \mathrm{Ma}$ (Dallmeyer et al., 1996). These age data speak for a rapid approach of the wedge-top Gemeric, Meliatic and Hronic nappes to the Fatric Basin southern margin until the Barremian. The Meliatic (and secondarily Hronic?) nappes may have initially yielded fine-grained $\mathrm{Cr}$-spinel and glaucophane detritus to unlithified Urgonian limestones of the inferred Fatric Basin southern margin from a relatively great distance or a relatively smooth relief. This situation was then dramatically changed by the Late Albian to Early Cenomanian at $\sim 110 / 105$ to $98 \mathrm{Ma}$ when the Gemeric, Meliatic (e.g., Kissová et al., 2005; Ivan et al., 2006) and most likely the Hronic (our recent study) nappe fragments closely approached this basin and added the pebble-to-boulder material to the Klape flysch sediments of this closing basin. Therefore, the Urgonian limestone pebble source at that time could also be the southern margin of the Fatric Basin because of its fault erosion during the basin transformation to a transpression-transtension-related flysch trough. Moreover, Jablonský et al. (2001) reported the presence of spinel detritus in the Valangian-Hauterivian strata of the Fatric Zliechov Succession. Plašienka et al. (2018) suggested the Urgonian limestone source from a Meliatic ephemeral basin. This model may contradict with the Meliatic nappe structure formation at that time (Putiš et al., 2014), and also missing such kind of facies in the Gosau-type sediments overlying the Gemeric and Silicic units (e.g., Hovorka et al., 1990).

\section{D2 AND D3 STAGES}

The D2 stage tectonic style of NW-vergent recumbent folds and the overthrust structures of the crystalline complexes including Upper Paleozoic and Mesozoic cover rocks predominates in the Selec Block (Putiš, 1983, 1986, 1991a). The D2 stage ( 95-85 Ma) exhumation, nappe structure formation, and northward thrusting of the Infratatric Inovec Nappe toward the Pieninic-Váhic ( South-Penninic) zone was followed by 85-65 Ma D3 supra-subductional extension. Thus, the near-surface Inovec Nappe posterior part could have supplied material to the Horné Belice wedge-top flysch basin located on the attenuated frontal Inovec Nappe-type basement. The Permian meta-sandstone illite-phengite to normal phengite (celadonite-rich muscovite) age of the Inovec Nappe at $\sim 86 \pm 2 \mathrm{Ma}$ (s. PI-14; Figs. 2 and 12D) then indicates re-opening of the ${ }^{40} \mathrm{Ar}-{ }^{39} \mathrm{Ar}$ system due to D2-D3 exhumation and thrusting (e.g., Villa, 2004; Villa et al., 2014).

The D3 stage Inovec Nappe supply of the Horné Belice flysch temporally overlaps with the commencement of the South-Penninic ( Pieninic-Váhic) subduction beneath the Klape-Manín-Inovec nappe system (Figs. 13 and 14). The sedimentary cycle of hemipelagic Upper Turonian-Lower Santonian Couches Rouges-type marls changed to Santonian to Maastrichtian Horné Belice syn-orogenic flysch sedimentation in a foreland wedge-top basin (e.g., DeCelles and Giles, 1996; Carrapa et al., 2016). This formed on a thinned Inovec Nappe-type basement in the inferred active continental margin ( the Humienec tectonic Slice in Fig. 13 herein and Putiš et al., 2006, 2008).

This time period overlaps with a sea-level rise in the Tethyan area (e.g., Lemoine, 2003; Cetean et al., 2011 and references therein) that is reflected in the deposition of hemipelagic red marls. The Schistes lustrés (SL) or Couches Rouges (CR)-type marls are characteristic on the distal margins and oceanic crust (Lemoine, 2003). These are usually Cenomanian to Maastrichtian, but up to the Paleocene-Eocene in some places. The SL and CR occur in all Penninic tectonic windows from Engadine to Rechnitz, and although they cannot precisely specify any stage in the Alpine geodynamic evolution, they are relative temporal markers in almost all the Alps and Corsican pelagic-hemipelagic domains (Lemoine, 2003). The finding of foraminifera Marginotruncana coronata Bolli specifies the age of the Infratatric C4 SL (after Lemoine, 2003) at Turonian to Santonian (Putiš et al., 2008).

A remnant of the Late Cretaceous distal Infratatric continental margin is inferred from the Humienec tectonic Slice of the Inovec Nappe which includes the infolded Upper Cretaceous sedimentary cover exposed in the footwall of the posterior part of the Inovec Nappe in the Považský Inovec Mts. (Figs. 2, 4, 6 , 13 and 14). Most importantly, the Humienec tectonic Slice, containing the micaschist basement and Permian to Upper Cretaceous cover rocks, indicates a partial D4 stage lower Infratatric nappe structure exposed in a tectonic window in the northern part of the Považský Inovec Mts. Selec Block (Figs. 2 and 14).

The ${ }^{40} \mathrm{Ar}-{ }^{39} \mathrm{Ar}$ ages from the Tatric Malé Karpaty, Tribeč, and the low-temperature blastomylonite zones of the western part of the Lúčanská Malá Fatra Mts. between $\sim 80$ and $70 \mathrm{Ma}$ (Hók et al., 2000; Král' et al., 2002; Putiš et al., 2009a) may constrain the postponed interaction of the North-Veporic-Tatric-Infratatric-PPKB units in a dextral transpressional system. The western "wedging-out" termination of the Tatric ribbon 
could then have enhanced lateral juxtaposition of these tectonic units (Figs. 1 and 13). Gosau-type restricted piggyback type of Late Cretaceous basins (e.g., Plašienka and Soták, 2015; Pelech et al., 2017a) could also indicate this transpression-transtensional event.

The pre-Eocene deformational effects at the lowest tectono-thermal conditions were also found at the Tatricum Nízke Tatry (Danišík et al., 2011), Žiar (Danišík et al., 2008) and Malá Fatra (Danišík et al., 2010) mountains, as documented by ZFT and/or zircon (U-Th)/He ages. These were resetted in the Vysoké Tatry Mts. during the Eocene (Anczkiewicz et al., 2015).

\section{D4 STAGE}

The common subvertical tectonic structures of the Inovec Nappe-type basement and infolded Upper Cretaceous sediments (Fig. 2), forming the frontal Infratatric Inovec Nappe Humienec tectonic Slice (Figs. 13 and 14), were traced by electric resistivity tomography to approximately $100 \mathrm{~m}$ depth (Pelech et al., 2017b).

The Inovec Nappe metamorphosed fragments, including the olistoliths of the Humienec Succession Upper Jurassic and Lower Cretaceous slates, occur in very low-anchi-metamorphosed (at $\sim 150-200^{\circ} \mathrm{C}$; Putiš et al., 2006, 2008) Upper Cretaceous flysch sediments (Kullmanová and Gašpariková, 1982). These are infolded to the partly re-built underground Albian-Early Cenomanian accretionary wedge with Inovec Nappe-type basement. The Late Maastrichtian?-Eocene late Meso-Alpine D4 deformation stage with NE-SW-striking thrust to the imbricated "klippen" tectonic structures predominates in the Považský Inovec Mts. Infratatric Selec Block (Figs. 1-7, 13 and 14).

The NW-SE-oriented reflection seismic profile 6HR (Fig. 3) reveals a detachment shear zone of the Infratatric Inovec Nappe at $\sim 6-7 \mathrm{~km}$ depth with underthrust PPKB units and deeper inferred fragments of the Pieninic-Váhic oceanic and/or continental crust and the PKB pre-Jurassic basement. The $\mathrm{MOHO}$ reflections at approximately $30 \mathrm{~km}$ depth generally indicate an attenuated crust. The concave shape of the $\mathrm{MOHO}$ and the higher crustal layers suggests the Neogene updoming, uplift and final exhumation of the Infratatric and underlying tectonic units.

The Eocene tectono-thermal overprinting in the D4 stage at $\sim 65-40$ Ma was dated on the newly formed celadonite-poor muscovite at the ${ }^{40} \mathrm{Ar}-{ }^{39} \mathrm{Ar}$ plateau age of $48 \pm 2 \mathrm{Ma}$ (Putiš et al., 2009a; s. PI-17HZ; Figs. 2 and 12E). This was established from hanging-wall granite blastomylonite of the Tatric Unit, and it is consistent with the ZFT 57-37 Ma ages (Fig. 8) determined from the inferred re-buried part of the Infratatric Inovec Nappe beneath the frontal Tatric Panská Javorina Nappe and the Paleogene sediments (Fig. 2). A similar whole-rock K-Ar age of $46 \pm 3$ Ma was also determined in a Permian basalt olistolith from the Upper Cretaceous flysch (Putiš et al., 2008). The Eocene Hrádok-Zlatníky thrust-fault is therefore the re-activated inferred Cretaceous thrust-fault of the Tatricum over the Infratatricum with the dextral strike-slip signatures concording with almost $\mathrm{E}-\mathrm{W}$-stretching blastomylonite lineations.

The Infratatric wedge formation in the Paleocene-Eocene was contemporaneous with the commenced closure of the Magura Basin in the OWC and the North-Penninic Ocean in the Alps, and with the onset of Central Carpathian Paleogene Basin (CCPB) sedimentation. A higher low-temperature history of the Inovec Nappe was determined by the relatively broad ZFT range of 57-37 Ma derived from the western, and likely deeper, NNE-SSW-striking steeply-dipping tectonic slices. This docu- ments sample residence, or passage through the ZFT partial annealing zone, during the Paleogene. It further indicates reheating of the Inovec Nappe to at least $180^{\circ} \mathrm{C}$ in the Eocene accretionary wedge caused by overloading of the Tatric Unit with the Mesozoic Fatric and Hronic nappes, and temporary burial beneath the CCPB sediments (Danišík et al., 2004, 2008, 2010; Anczkiewicz et al., 2015). The Eocene exhumation was followed by final cooling just below $100^{\circ} \mathrm{C}$ during the Miocene orogen parallel extension derived from the 21-13 Ma AFT ages (Kováč et al., 1994; Danišík et al., 2004; Králiková et al., 2016).

The presence of the Cretaceous and Paleocene-Eocene ZFT populations in our four samples (Tables 1 and 2) clearly documents the Alpine overprint in the Selec Block expected from geological observations, metamorphic assemblages and ${ }^{40} \mathrm{Ar}-{ }^{39} \mathrm{Ar}$ data; and it therefore contradicts the Alpine tectogenesis absence in the Infratatric Selec Block, reported by Králiková et al. (2016).

The exhumation of the Infratatricum and the northern border of the Tatricum from $\sim 10-5 \mathrm{~km}$ depth occurred in the footwall of the higher Fatric and Hronic nappes. This process is supported by the approximately $48 \mathrm{Ma}$ temporally constrained exhumation character of the Eocene Hrádok-Zlatníky thrust fault which is partly covered by the fragments of the Fatric (Krížna) and Hronic (Choč) nappes (Figs. 2 and 13).

\section{CONCLUSIONS - EVOLUTIONARY MODEL OF THE INFRATATRICUM}

In conclusion, the following updated Infratatricum evolutionary model is presented (Fig. 14):

1. In the pre-Late Cretaceous, a distal "pre-Infratatric" northern passive continental margin with the Middle Jurassic to Lower Cretaceous Humienec Succession was facing the Pieninic-Váhic ( South-Penninic) Basin Realm. The basement of the Humienec Succession was formed by the syn-rift Lower Jurassic and the pre-rift Triassic, Permian and Upper Carboniferous sedimentary and volcanic rocks on the attenuated Variscan crystalline basement. The closure of the Jurassic-Early Cretaceous basin was followed by the D1 stage late Early Cretaceous tectono-metamorphic event in an Albian-Early Cenomanian accretionary wedge ( 115-95 Ma; Figs. 8, 12A-C and 14). A much more effective tectono-thermal D1 stage impact was registered in the Infratatric Selec Block (pronounced higher- to middle anchi-metamorphism) in comparison with the Tatric Bojná Block hanging-wall blastomylonitization.

2. The Inovec Nappe developed from the Albian-Early Cenomanian orogenic wedge in front of the Tatricum. This nappe was thrust onto an inferred transitional continental/oceanic crust adjoining the South-Penninic (Pieninic-Váhic) Ocean and, in turn, overthrust by the Fatric Manín-Klape and Hronic nappes during the D2 stage at $\sim 95-90 / 85 \mathrm{Ma}$. The Ar-Ar age of $86 \pm 2 \mathrm{Ma}$ from a Permian meta-sandstone of the Inovec Nappe can be related to this event (Fig. 12D) followed by the deposition of red marls in the Late Turonian to Early Santonian (Fig. 14).

3. The Late Cretaceous southward subduction of the inferred Pieninic-Váhic crust caused further differentiation into: (1) a distal "Infratatric" active continental margin in the D3 stage at $\sim 85-65$ Ma defined by the upper Santonian-Maastrichtian Horné Belice flysch succession of a foreland basin located on the thinned Infratatric 
Inovec Nappe-type basement. In support, Kullmanová and Gašpariková (1982) report a similar Upper Cretaceous sedimentary cover in the Manín Unit Soblahov borehole, and (2) a hinterland Tatric domain with the piggy-back basin Hubina Formation (Pelech et al., 2017a) located on a thicker basement. The input of metamorphosed material from the posterior part of the Infratatric Inovec Nappe to the foreland Horné Belice Basin during the D3 stage is temporally consistent with the ZFT ages of $69 \pm 8 \mathrm{Ma}$ and $74 \pm 6 \mathrm{Ma}$ reported from the Považský Inovec Mts. Tatric Bojná Block (Kováč et al., 1994; Králiková et al., 2016).

4. Closure of the Late Cretaceous Horné Belice Basin led to the Infratatricum becoming part of a fold-thrust to steeply-dipping transpressional klippen-type structure with incorporated Upper Cretaceous sediments in the Humienec tectonic Slice overriden by the posterior part of the Inovec Nappe (Figs. 2 and 14). The Eocene reactivation age of $\sim 48 \pm 2 \mathrm{Ma}$ approximates the D4 stage of $\sim 65-40 \mathrm{Ma}$ (Figs. 12E, 13 and 14). This age for the inferred Late Cretaceous tectonic contact of the Tatricum and Infratatricum Hrádok-Zlatníky transpressional thrust-fault suggests new Paleocene-Eocene accretionary wedge formation in the Tatric foreland. Thus, the Hrádok-Zlatníky thrust-fault formed an Eocene micro-plate boundary because the Infratatricum became part of the lower plate PPKB and PKB units overriden by the Tatricum and higher tectonic units (see also the $6 \mathrm{HR}$ seismic profile in Fig. 3). The Infratatricum is finally a Cenozoic tectonic unit located north of the composite Late Jurassic-Early Cretaceous Neotethyan accretionary wedge (Meliatic-Gemeric-South-Veporic; 150-100 $\mathrm{Ma}$ ) and part of the Southern Atlantic Tethys orogenic wedge (N-Veporic-Tatric-Infratatric; 100-50 Ma).
5. The reheating and exhumation cooling of the Infratatricum in the footwall of the Tatricum northern edge and accompanying Paleogene sediments is documented by ZFT ages of 57-37 Ma. Final exhumation and cooling occurred in the Miocene due to orogen-parallel extension (21-13 Ma AFT ages; Danišík et al., 2004; Králiková et al., 2016).

6) The $6 \mathrm{HR}$ reflection seismic profile shows that the approximately $40^{\circ}$ dipping thrust-fault of the Infratatricum over the foreland PPKB units transforms to sub-horizontal and then concave at 6-7 km depth (Fig. 3). The sub-horizontal reflection zones may indicate rather shallow underthrusting of the foreland crustal sheets. Finally, ZFT data confirms that the observed concavity is related to the exhumation of the Infratatric and underlying decoupled PPKB-PKB fragments in the footwall of the Tatricum and Paleogene sediments in the late Eocene, and AFT data verifies the Miocene exhumation and cooling.

Acknowledgement. The authors express their gratitude to D. Plašienka and unknown referee for constructive reviews of this paper. R. Marshall and K. Leszczyński are thanked for language review. A. Wysocka is thanked for editorial handling of this paper. APVV-15-0050 and VEGA 1/0079/15 grants (M.P.) are greatly acknowledged. M.D. was supported by the AuScope NCRIS2 program, Australian Scientific Instruments Pty Ltd., Australian Research Council (ARC) Discovery funding scheme (DP160102427), Curtin Research Fellowship, and by the German Science Foundation (DFG), project KU 1298/3. M.D. thanks I. Dunkl and W. Frisch for training in the fission track dating method, interpretation of the data and access to the FT laboratory in Tübingen.

\section{REFERENCES}

Anczkiewicz, A.A., Danišík, M., Środoń, J., 2015. Multiple low-temperature thermochronology constraints on exhumation of the Tatra Mountains: new implication for the complex evolution of the Western Carpathians in the Cenozoic. Tectonics, 34 2296-2317.

Armbruster, T., Bonazzi, P., Akasaka, M., Bermanec, V., Chopin, Ch., Gieré, R., Heuss-Assbichler, S., Liebscher, A., Menchetti, S., Pan, Y., Pasero, M., 2006. Recommended nomenclature of epidote-group minerals. European Journal of Mineralogy, 18: 551-567.

Aubrecht, R., Méres, Š., Sýkora, M., Mikuš, T., 2009. Provenance of the detrital garnets and spinels from the Albian sediments of the Czorsztyn Unit (Pieniny Klippen Belt, Western Carpathians, Slovakia). Geologica Carpathica, 60: 463-483.

Bezák, V., 1994. Proposal of the new division of the West Carpathian crystalline based on the Hercynian tectonic building reconstruction (in Slovak with English summary). Mineralia Slovaca, 26: 1-6.

Birkenmajer, K., Kozur, H., Mock, R., 1990. Exotic Triassic pelagic limestone pebbles from the Pieniny Klippen Belt of Poland: a further evidence for early Mesozoic rifting in West Carpathians. Annales Societatis Geologorum Poloniae, 60: 3-44.

Brandon, M.T., 2004. The Cascadia subduction wedge: the role of accretion, uplift, and erosion. In: Earth Structure: an Introduction to Structural Geology and Tectonicsvan (eds. B.A. der Pluijm and S. Marshak): 566-574. WCB/McGrawHill Press.

Buday, T., ed., Cambel, B., Mahel', M., Brestenská, E., Kamenický, J., Kullmann, E., Matějka, A., Salaj, J., Za ko, M., 1962. Explanations to Geological Map of Czechoslovakia
1:200 000, Sheet Vienna - Bratislava. Geological Survey of Slovak Republic.

Buday, T., ed., Benešová, E., Březina, J., Cicha, I., Čtyroký, P., Dornič, J., Dvořák, J., Eliáš, M., Hanzlíková, E., Jendrejáková, O., Kačura, G., Kamenický, J., Kheil, J., Köhler, E., Kullmanová, A., Mahel', M., Matějka, A., Paulík, J., Salaj, J., Scheibner, E., Scheibnerová, V., Stehlík, O., Urbánek, L., Vavř́ínová, M., Zelman, J., 1963. Explanations to Geological Map of Czechoslovakia 1:200 000, Sheet Gotwaldov. Czechoslovak Academy of Sciences, Prague.

Carrapa, B., Di Giulio, A., Mancin, N., Stockli, D., Fantoni, R., Hughes, A., Gupta, S., 2016. Tectonic significance of Cenozoic exhumation and foreland basin evolution in the Western Alps. Tectonics, 35, doi: 10.1002/2016TC004132

Cathelineau, M., 1988. Cation site occupancy in chlorites and illites as function of temperature. Clay Minerals, 23: 471-485.

Cetean, C.G., Bălc R., Kaminski, M.A., Filipescu, S., 2011. Integrated biostratigraphy and palaeoenvironments of an upper Santonian-upper Campanian succession from the southern part of the Eastern Carpathians, Romania. Cretaceous Research, 32: 575-590.

Connolly, J.A.D., 2005. Computation of phase-equilibria by linear programming: a tool for geodynamic modeling and its application to subduction zone decarbonation. Earth and Planetary Science Letters, 236: 524-541.

Connolly, J.A.D., Petrini, K., 2002. An automated strategy for calculation of phase diagram sections and retrieval of rock properties as a function of physical conditions. Journal of Metamorphic Petrology, 20: 697-708. 
Csontos, L., Vörös, A., 2004. Mesozoic plate-tectonic reconstruction of the Carpathian region. Palaeogeography, Palaeoclimatology, Palaeoecology, 210: 1-56.

Dallmeyer, R.D., Neubauer, F., Handler, R., Fritz, H., Müller, W. Pana, D., Putiš, M., 1996. Tectonothermal evolution of the internal Alps and Carpathians: Evidence from ${ }^{40} \mathrm{Ar} /{ }^{39} \mathrm{Ar}$ mineral and whole-rock data. Eclogae Geologicae Helvetiae, 89: 203-227.

Dal Piaz, G.V., Martin, S., Villa, I., Gosso, G., Marschalko, R. 1995. Late Jurassic blueschist facies pebbles from the western Carpathian orogenic wedge and paleostructural implications for western Tethys evolution. Tectonics, 14: 874-885.

Danišík, M., Dunkl, I., Putiš, M., Frisch, W., Král', J., 2004. Tertiary burial and exhumation history of basement highs along the NW margin of the Pannonian Basin - an apatite fission track study. Austrian Journal of Earth Sciences, 95/96: 60-70.

Danišík, M., Dunkl, I., Putiš, M., Frisch, W., Král', J., 2005. Erratum to „Tertiary burial and exhumation history of basement highs along the NW margin of the Pannonian Basin - an apatite fission track study". Austrian Journal of Earth Sciences, 97: 94.

Danišík, M., Kuhlemann, J., Dunkl, I., Szekely, B., Frisch, W., 2007. Burial and exhumation of Corsica (France) in the light of fission track data. Tectonics, 26: 1-24.

Danišík, M., Kohút, M., Dunkl, I., Frisch, W., 2008. Thermal evolution of the Žiar Mountains basement (Inner Western Carpathians, Slovakia) constrained by fission track data. Geologica Carpathica, 59: 19-30.

Danišík, M., Kohút, M., Broska, I., Frisch, W., 2010. Thermal evolution of the Malá Fatra Mountains (Central Western Carpathians): insights from zircon and apatite fission track thermochronology. Geologica Carpathica, 61: 19-27.

Danišík, M., Kadlec, J., Glotzbach, C., Weisheit, A., Dunkl, I., Kohút, M., Evans, N.J., Orvošová, M., McDonald, B.J., 2011. Tracing metamorphism, exhumation and topographic evolution in orogenic belts by multiple thermochronology: a case study from the Nízke Tatry Mts., Western Carpathians. Swiss Journal of Geosciences, 104: 285-298.

Dantzig, G., 2016. Linear Programming and Extensions. Princeton University Press.

DeCelles, P.G., Giles, K.A., 1996. Foreland basin sytems. Basin Research, 8: 105-123.

Dumitru, T.A., 1993. A new computer-automated microscope stage system for fission-track analysis. Nuclear Tracks and Radiation Measurements, 21: 575-580.

Dunkl, I., 2002. TRACKKEY: a Windows program for calculation and graphical presentation of fission track data. Computers \& Geosciences, 28: 3-12.

DunkI, I., Székely, B., 2003. Component Analysis with Visualization of Fitting-Popshare, a Freeware Program for Evaluation of Mixed Geochronological Data. EGS-AGU-EUG Joint Assembly.

Faryad, S.W., 1995. Phase petrology and P-T conditions of mafic blueschists from the Meliata Unit, Western Carpathians, Slovakia. Journal of Metamorphic Geology, 13: 701-714.

Faryad, S.W., 1997. Petrological model for blueschist facies metamorphism in the Pieniny Klippen Belt. In: Geological Evolution of the Western Carpathians (eds. P. Grecula, D. Hovorka and M. Putiš): 155-162. Mineralia Slovaca - Monograph., Bratislava.

Faryad, S.W., Frank, W., 2011. Textural and age relations of polymetamorphic rocks in the HP Meliata Unit (Western Carpathians). Journal of Asian Earth Sciences, 42: 111-122.

Faryad, S.W., Henjes-Kunst, F., 1997. Petrological and K-Ar and ${ }^{40} \mathrm{Ar}-{ }^{39} \mathrm{Ar}$ age constraints for the tectonothermal evolution of the high-pressure Meliata unit, Western Carpathians (Slovakia) Tectonophysics, 280: 141-156.

Foland, K.A., Hubacher, F.A., Arehart, G.B., 1992. ${ }^{40} \mathrm{Ar} /{ }^{39} \mathrm{Ar}$ dating of very fine-grained samples: an encapsulated-vial procedure to overcome the problem of ${ }^{39} \mathrm{Ar}$ recoil loss. Chemical Geology, 102: 269-276.

Galbraith, R.F., Laslett, G.M., 1993. Statistical models for mixed fission track ages. Nuclear Tracks and Radiation Measurements, 21: 459-470.

Gawlick, H.-J., Missoni, S., 2015. Middle Triassic radiolarite pebbles in the Middle Jurassic Hallstatt Mélange of the Eastern Alps: Implications for Triassic-Jurassic geodynamic and palaeogeographic reconstructions of the western Tethyan realm. Facies: 61: 13, doi: 10.1007/s10347-015-0439-3
Gleadow, A.J.W., 1981. Fission-track dating methods: what are the real alternatives? Nuclear Tracks, 5: 3-14.

Halama, R., Konrad-Schmolke, M., Sudo, M., Marschall, H.R., Wiedenbeck, M., 2014. Effects of fluid-rock interaction on ${ }^{40} \mathrm{Ar} /{ }^{39} \mathrm{Ar}$ geochronology in high-pressure rocks (Sesia-Lanzo Zone, Western Alps). Geochimica et Cosmochimica Acta, 126: 475-494.

Havrila, M., Vaškovský, I., 1983. Explanations to Geological Map of Mesozoicum in southdern part of Považský Inovec Mts. Manuscript. Geofond, Bratislava.

Hawthorne, F.C., Oberti, R., Harlow, G.E., Maresch, W.V., Martin R.F., Schumacher J.C., Welch, M.D., 2012. Nomenclature of the amphibole supergroup. American Mineralogist, 97: 2031-2048.

Hók, J., Siman, P., Frank, W., Král', J., Kotulová, J., Rakús, M. 2000. Origin and exhumation of mylonites in the Lúčanská Malá Fatra Mts., (the Western Carpathians). Slovak Geological Magazine, 6: 325-334.

Holland, T.J.B., Powell, R., 1998, updated 2002. An internally consistent thermodynamic dataset for phases of petrological interest. Journal of Metamorphic Geology, 16: 309-343.

Hovorka, D., Ivan, P., Mock, R., Rozložník, L., Spišiak, J., 1990. Sediments of Gosau type near the Dobšiná Ice Cave: ideas for their non-traditional interpretation (in Slovak with English summary). Mineralia Slovaca, 22: 519-525.

Hunziker, J.C., Frey, M., Claber, N., Kallmeyer, R.D., Friedrichsen, H., Flehming, W., Hochstrasser, K., Roggwiller, P., Schwander, H., 1986. The evolution of illite to muscovite: mineralogical and isotope data from the Glarus Alps, Schwitzerland. Contributions to Mineralogy and Petrology, 92: 157-180.

Hurai, V., Paquette, J.-L., Lexa, O., Konečný, P., Dianiška, I., 2015. U-Pb-Th geochronology of monazite and zircon in albitite metasomatites of the Rožňava-Nadabula ore field (Western Carpathians, Slovakia): implications for the origin of hydrothermal polymetallic siderite veins. Mineralogy and Petrology, 109: 519-530.

Hurford, A.J., Green, P.F., 1983. The zeta age calibration of fission-track dating. Chemical Geology, 41: 285-317.

Ivan, P., Sýkora, M., 1993. Finding of glaucophane-bearing rocks in Cretaceous conglomerates from Jasenov (Krížna nappe, Eastern Slovakia) (in Slovak with English abstract). Mineralia Slovaca, 25: 29-33.

Ivan, P., Sýkora, M., Demko, R., 2006. Blueschists in the Cretaceous exotic conglomerates of the Klape Unit (Pieniny Klippen Belt, Western Carpathians): their genetic types an implications for source area. Geologia, 32: 47-63.

Ivanička, J., Hók, J., Polák, M., Határ, J., Vozár, J., Nagy, A., Fordinál, K., Pristaš, J., Konečný, V., Šimon, L., Kováčik, M., Vozárová, A., Fejdiová, O., Marcin, D., Liščák, P., Macko, A., Lanc, J., Šantavý, J., Szalaiová, V., 1998. Explanations to the Geological Map of the Tribeč Mts. 1:50 000 (in Slovak with English summary). Geological Survey of Slovak Republic, Bratislava

Ivanička, J., Havrila, M., Kohút, M., eds., Kováčik, M., Madarás, J., Olšavský, M., Hók, J., Polák, M., Filo, I., Elečko, M., Fordinál, K., Maglay, J., Pristaš, J., Buček, S., Šimon, L., 2007. Geological map of the Považský Inovec Mts. and SE part of the Trenčianska kotlina Depression 1:50 000 (in Slovak with English summary). State Geological Institute of Dionýz Štúr, Bratislava.

Ivanička, J., Kohút, M., eds., Havrila, M., Olšavský, M., Hók, J., Kováčik, M., Madarás, J., Polák, M., Rakús, M., Filo, I. Elečko, M., Fordinál, K., Maglay, J., Pristaš, J., Buček, S. Šimon, L., Kubeš, P., Scherer, S., Zuberec, J., Dananaj, I., Klukanová, A., 2011. Explanations to the Geological Map of the Region of the Považský Inovec Mts., and SE part of the Trenčianska kotlina Depression 1:50 000 (in Slovak with English summary). State Geological Institute of Dionýz Štúr, Bratislava.

Jablonský, J., Sýkora, M., Aubrecht, R., 2001. Detritic Cr-spinels in Mesozoic sedimentary rocks of the Western Carpathians (overview of the latest knowledge) (in Slovak with English summary). Mineralia Slovaca, 33: 487-498.

Jowett, E., 1991. Fitting iron and magnesium into the hydrothermal chlorite geothermometer: GAC/MAC/SEG. Presented at the 
Joint Annual Meeting. Toronto, May 27-29. Program with Abstracts.

Kamenický, J., 1956. Geological report on mapping works in northern part of the Považský Inovec Mts. crystalline complex (in Slovak). Geologické Práce, Zprávy, 8: 110-124

Kissová, D., Dunkl, I., Plašienka, D., Frisch, W., Marschalko, R. 2005. The Pieninic exotic cordillera (Andrusov Ridge) revisited new zircon FT ages of granite pebbles from Cretaceous conglomerates of the Pieniny Klippen Belt (Western Carpathians, Slovakia). Slovak Geological Magazine, 11: 17-28.

Korikovsky, S.P., Putiš, M., 1999. Evolution of authigenic and detrital K-micas at the boundary between anchimetamorphism and low-temperature metamorphism during the Cretaceous tectono-metamorphic cycle in the Western Carpathians. Petrology, 7: 364-382.

Korikovsky, S.P., Putiš, M., Ďurovič, V., Zakariadze, G.S., 1995 Alpine anchimetamorphism of the Infratatricum cover, Western Carpathians: composition of authigenic and detrital muscovite phengite as an indicator of the metamorphic grade. Petrology, 3 525-538.

Kováč, M., Král', J., Márton, E., Plašienka, D., Uher, P., 1994. Alpine uplift history of the Central Western Carpathians: geochronological, paleomagnetic, sedimentary and structural data. Geologica Carpathica, 45: 83-96.

Kozur, H., 1991. The evolution of the Meliata-Hallstatt Ocean and its significance forthe early evolution of the Eastern Alps and Western Carpathians. Palaeogeography, Palaeoclimatology, Palaeoecology, 87: 109-135.

Král', J., Hók, J., Frank, W., Siman, P., Liščák, P., Jánová, V. 2002. Shear deformation in granodiorite: structural, ${ }^{40} \mathrm{Ar} /{ }^{39} \mathrm{Ar}$ and geotechnical data (Tribeč Mts., Western Carpathians). Slovak Geological Magazine, 8: 235-246.

Král', J., Hók, J., Bachliński, R., Ivanička, J., 2013. Age of diaphtoritic rocks from the Považský Inovec Mts.: Rb-Sr and ${ }^{40} \mathrm{Ar} /{ }^{39} \mathrm{Ar}$ mineral data (Western Carpathians). Acta Geologica Slovaca, 5: 195-210.

Králiková, S., Vojtko, R., Hók, J., Fügenschuh, B., Kováč, M., 2016. Low-temperature constraints on the Alpine thermal evolution of the Western Carpathian basement rock complexes. Journal of Structural Geology, 91: 144-160.

Krist, E., Korikovsky, S.P., Putiš, M., Janák, M., Faryad, S.W., 1992. Geology and Petrology of Metamorphic Rocks of the Western Carpathian Crystalline Complexes. Comenius University Press, Bratislava.

Kullmanová, A., Gašpariková, V., 1982. Upper Cretaceous sediments in northern part of Považský Inovec Mts. (in Slovak with English summary). Geologické Práce, Správy, 78: 85-95.

Lemoine, M., 2003. Schistes lustrés from Corsica to Hungary: back to the original sediments and tentative dating of partly azoic metasediments. Bulletin de la Société Géologique de France, 174: 197-209.

Leško, B., Šutora, A., Putiš, M., 1988. Geology of the Považský Inovec Horst based on geophysical investigation. Geologický zborník Geologica Carpathica, 39: 195-216.

Lexa, J., Bezák, V., Elečko, M., Mello, J., Polák, M., Potfaj, M., Vozár, J., eds., 2000. Geological map of Western Carpathians and adjacent areas 1:500 000. Ministry of the Environment of Slovak Republic. Geological Survey of Slovak Republic Bratislava.

Mahel', M., 1981. Island character of Klippen Belt; Váhikum - continuation of southern Pennicum in West Carpathians. Geologicky zborník Geologica Carpathica, 32: 293-305.

Mahel', M., 1986. Geologická stavba Československých Karpát. 1 Paleoalpínske jednotky (in Slovak). Veda, Bratislava.

Marschalko, R., Rakús, M., 1997. Development of the Cretaceous flysch in the Klape unit and the recyclicity problem of the clastic material. In: Alpine Evolution of the Western Carpathians and Related Areas (eds. D. Plašienka, J. Hók, J. Vozár and M Elečko): 71-78. Internat. Conference, Introductory Articles to the Excursion. Geol. Survey Slovak Rep. Bratislava.

Massonne, H.-J., Schreyer, W., 1987. Phengite geobarometry based on the limiting assemblage with K-feldspar, phlogopite, and quartz. Contributions to Mineralogy and Petrology, 96: 212-224.
Mišík, M., 1979. Pieniny Klippen Belt and the global tectonics model. In: Czechoslovak Geology and Global Tectonics (eds. M. Mahel' and P. Reichwalder): 89-101. Bratislava, Veda Publishers.

Mišík, M., Marschalko, R., 1988. Exotic conglomerates in flysch sequences: examples from the West Carpathians. Mémoires de la Société Géologique de France, Paris, Nou velle Série, 154: 95-115.

Mišík, M., Sýkora, M., 1981. Der pieninische exotische Rücken, rekonstruiert aus Geröllen karbonatischer Gesteine kretazischer Konglomerate der Klippenzone und der ManínEinheit (in Slovak with German summary). Západné Karpaty, sér. geológia, 7: 7-111.

Mišík, M., Jablonský, J., Mock, R., Sýkora, M., 1981. Konglomerate mit exotischen Material in dem Alb der Zentralen Westkarpathen - paläogeographische and tektonische Interpretation. Acta Geologica et Geographica Universitatis Comenianae, 37: 5-55.

Neubauer, F., Genser, J., Handler, R., 2000. The Eastern Alps: result of two-stage collision process. Mitteilungen der Österreichischen Geologischen Gesellschaft, 92: 117-134.

Olšavský, M., 2008. Lithostratigraphy and sedimentogenesis of Upper Paleozoic strata in northern part of the Považský Inovec Mts. (in Slovak with English summary). Mineralia Slovaca, 40 $1-16$.

Oszczypko, N., Ślączka, A., Oszczypko-Clowes, M., Olszewska, B., 2015. Where was the Magura Ocean? Acta Geologica Polonica, 65: 319-344.

Pelech, O., Hók, J., Havrila, M., Pešková l., 2016a. Structural position of the Upper Cretaceous sediments in the Považský Inovec Mts. (Western Carpathians). Acta Geologica Slovaca, 8: 43-58.

Pelech, O., Józsa, Š., Kohút, M., Plašienka, D., Hók, J., Soták, J., 2016b. Structural, biostratigraphic and petrographic evaluation of the Upper Cretaceous red marlstones and underlying granitoids from the borehole HPJ-1 Jašter near Hlohovec (Považský Inovec Mts., Slovakia). Acta Geologica Slovaca, 8: 27-42.

Pelech, O., Hók, J., Józsa, Š., 2017a. Turonian-Santonian sediments in the Tatricum of the Považský Inovec Mts. (Internal Western Carpathians, Slovakia). Austrian Journal of Earth Sciences, 110: 21-35.

Pelech, O., Kušnirák, D., Bošanský, M., Dostál, I., Putiška, R., Hók, J., 2017b. The resistivity image of the Upper Cretaceous Horné Belice Group: a case study from the Hranty section (Považský Inovec Mts., Western Carpathians). Contributions to Geophysics and Geodesy, 47: 23-38.

Plašienka, D., 1995. Passive and active margin history of the northern Tatricum (Western Carpathians, Slovakia). Geologische Rundschau, 84: 748-760.

Plašienka, D., 2012. Jurassic syn-rift and Cretaceous synorogenic, coarse-grained deposits related to opening and closure of the Vahic (South Penninic) Ocean in the Western Carpathians - an overview. Geological Quarterly, 56 (4): 601-628.

Plašienka, D., 2018. Continuity and episodicity in the Early Alpine tectonic evolution of the Western Carpathians: how large-scale processes are expressed by the orogenic architecture and rock record data. Tectonics, 37: 2029-2079.

Plašienka, D., Soták, J., 2015. Evolution of Upper Cretaceous-Paleogene synorogenic basins in the Pieniny Klippen Belt and adjacent zones (Western Carpathians, Slovakia): tectonic controls over a growing orogenic wedge. Annales Societatis Geologorum Poloniae, 85: 43-76.

Plašienka, D., Marschalko, R., Soták, J., Peterčáková, M., Uher P., 1994. Origin and structural position of Upper Cretaceous sediments in northern Považský Inovec Mts. (in Slovak with English summary). Part One: Lithostratigraphy and sedimentology. Mineralia Slovaca, 26: 311-334.

Plašienka, D., Grecula, P., Putiš, M., Kováč, M., Hovorka, D., 1997. Evolution and structure of the Western Carpathians: an overview. In: Geological Evolution of the Western Carpathians (eds. P. Grecula, D. Hovorka and M. Putiš). Mineralia Slovaca Monograph., Bratislava.

Plašienka, D., Méres, Š., Ivan, P., Sýkora, M., Soták, J., Lačný, A. Aubrecht, R., Bellová, S., Potočný, T., 2018. Meliatic blueschists and their detritus in Cretaceous sediments: new data con- 
straining tectonic evolution of the West Carpathians. Swiss Journal of Geosciences, doi: 10.1007/s00015-018-0330-7

Platt, J.P., 1993. Exhumation of high-pressure rocks: a review of concepts and processes. Terra Nova, 5: 119-133.

Putiš, M., 1983. Outline of geological-structural development of the crystalline complex and envelope Paleozoic of the Považský Inovec Mts. Geologický zborník Geologica Carpathica, 34: 457-482.

Putiš, M., 1986. Upper Palaeozoic complex of the Považský Inovec Mts. (in Slovak with English summary). Geologické Práce, Správy, 84: 65-83.

Putiš, M., 1987. Geology and tectonics of southwestern and northern part of the Malé Karpaty Mts. crystalline complex (in Slovak with English summary). Mineralia Slovaca, 19: 135-157.

Putiš, M., 1991a. Tectonic styles and Late Variscan-Alpine evolution of the Tatric-Veporic crystalline basement in the Western Carpathians. Zentralblatt für Geologie und Paläontologie, 1 181-204.

Putiš, M., 1991b. Geology and petrotectonics of some shear zones in the West Carpathian crystalline complexes. Mineralia Slovaca, 23, Newsletter 3: 459-473.

Putiš, M., 1992. Variscan and Alpidic nappe structures of the Western Carpathian crystalline basement. Geologica Carpathica, $\mathbf{4 3}$ : 369-380.

Putiš, M., Tomek, Č., 2016. Metamorphosed sources of the Late Cretaceous flysch in the Infratatric Albian-Eocene accretionary wedge: impact on large-scale tectonics. 10th ESSEWECA Conference, Abstract Book, Bratislava, Slovakia: 80-82.

Putiš, M., Németh, Z., Unzog, W., Wallbrecher, E., 1999. The quartz and calcite X-ray texture goniometer patterns from the Western Carpathians Cretaceous ductile shear zones used as kinematic indicators. Geologica Carpathica, Spec. Issue, $\mathbf{5 0}$ 165-169.

Putiš, M., Sulák, M., Gawlick, H.J., Bojar, A.V., 2006. Infratatricum of the Považský Inovec Mts. (Western Carpathians): Geodynamic evolution of continental margin in the vicinity of the oceanic Penninicum (in Slovak with English summary) Mineralia Slovaca, 38: 7-28.

Putiš, M., Gawlick, H.J., Frisch, W., Sulák, M., 2008. Cretaceous transformation from passive to active continental margin in the Western Carpathians as indicated by the sedimentary record in the Infratatric Unit. International Journal of Earth Sciences (Geologische Rundschau), 97: 799-819.

Putiš, M., Frank, W., Plašienka, D., Siman, P., Sulák, M., Biroň, A., 2009a. Progradation of the Alpidic Central Western Carpathians orogenic wedge related to two subductions: constrained by ${ }^{40} \mathrm{Ar} /{ }^{39} \mathrm{Ar}$ ages of white micas. Geodinamica Acta 22: $31-56$.

Putiš, M., Ivan, P., Kohút, M., Spišiak, J., Siman, P., Radvanec M., Uher, P., Sergeev, S., Larionov, A., Méres, Š., Demko, R., Ondrejka, M., 2009b. Metaigneous rocks of the WestCarpathian basement, Slovakia: indicators of Early Paleozoic extension and shortening events. Bulletin de la Societe Geologique de France, 180: 461-471.

Putiš, M., Danišík, M., Ružička, P., Schmiedt, I., 2014. Constraining exhumation pathway in an accretionary wedge by (U-Th)/He thermochronology - case study on Meliatic nappes in the Western Carpathians. Journal of Geodynamics, 81: 80-90.

Putiš, M., Yang, Y.-H., Koppa, M., Dyda, M., Šmál, P., 2015. U/Pb LA-ICP-MS age of metamorphic-metasomatic perovskite from serpentinized harzburgite in the Meliata unit at Dobšiná, Slovakia: Time constraints of fluid-rock interaction in an accretionary wedge. Acta Geologica Slovaca, 7: 63-71.

Putiš, M., Ružička, P., Ling, X., Nemec, O., 2016. U/Pb SIMS zircon dating of a rhyolite intercalation in Permian siliciclastics as well as a rhyodacite dyke in micaschists (Infratatricum, W. Carpathians). Mineralia Slovaca, 48: 135-144

Putiš, M., Li, X.-H., Ondrejka, M., Siman, P., Németh, Z., Koller, F., Nemec, O., Ružička, P., 2017. The Eoalpine Orogen evolution from Permian-Triassic extension to Jurassic-Eocene accretion determined by Zrn U/Pb SIMS, microprobe Mnz and WhM Ar/Ar ages in the Western Carpathians and the Eastern Alps. 13th Workshop on Alpine Geological Studies - Émile Ar- gand Conference (EGU Series) Zlatibor, Serbia: 89-90. Abstract Book, University of Belgrade.

Putiš, M., Németh, Z., Li, X.-H., Yang, Y.-H., Li, Q.-L., Ling, X., Nemec, O., Ondrejka, M., 2018a. Prototethys, Paleotethys and Neotethys remnants in the Western Carpathians constrained by SIMS and LA-ICP-MS U-Pb zircon ages. Geophysical Research Abstracts, 20, EGU2018-1678, Copernicus Göttingen.

Putiš, M., Plašienka, D., Danišík, M., Tomek, Č., 2018b Infratatricum of the Inner Western Carpathians: coupled Albian-Eocene accretionary wedge exposed in a Miocene tectonic window. Geology, Geophysics and Environment, 44: 187-189

Rakús, M., Marschalko, R., 1997. Position of the Manín, Drietoma and Klape units at the boundary of the Central and Outer Carpathians. In: Alpine Evolution of the Western Carpathians and Related Areas (eds. D. Plašienka, J. Hók, J. Vozár and M. Elečko): 79-97. Inernational. Conference, Introductory Articles to the Excursion. Geol. Survey Slovak Rep. Bratislava.

Scharf, A., Handy, M.R., Schmid, S.M., Favaro, S., Sudo, M., Schuster, R., Hammerschmidt, K., 2016. Grain-size effects on the closure temperature of white mica in a crustal-scale extensional shear zone - implications of in-situ ${ }^{40} \mathrm{Ar} /{ }^{39} \mathrm{Ar}$ laser-ablation of white mica for dating shearing and cooling (Tauern Window, Eastern Alps). Tectonophysics, 674: 210-226.

Schmid, S.M., Bernoulli, D., Fügenschuh, B., Matenco, L., Schefer, S., Schuster, R., Tischler, M., Ustaszewski, K., 2008. The Alpine-Carpathian-Dinaridic orogenic system: correlation and evolution of tectonic units. Swiss Journal of Geosciences, 101: 139-183.

Sperner, B., Pfänder, J.A., Scherer, A., Ratschbacher, L., 2010 Ar-Ar dating of biotite - grain size dependent ages and possible interpretations. Geophysical Research Abstracts, 12: EGU2010-13708.

Sulák, M., Kaindl, R., Putiš, M., Sitek, J., Krenn, K., Tóth, I., 2009. Chemical and spectroscopic characteristics of potassium white micas related to polystage evolution of the Central Western Carpathians orogenic wedge. Lithos, 113: 709-730.

Šímová, M., 1985. Magmatic roks from the Cretaceous conglomerates of the western part of the Klippen and Manín Belts (in Slovak with English summary). Západné Karpaty, sér. Mineralógia, petrografia, geochémia, metalogenéza, 10: 9-110.

Tischendorf, G., Förster, H.J., Gottesmann, B., Rieder, M., 2007 True and brittle micas: composition and solid-solution series. Mineralogical Magazine, 71: 285-320.

Tollmann, A., 1980. Geology and tectonics of the Eastern Alps (middle sector). Abhandlungen der Geologischen Bundesanstalt, 34: 197-255.

Tomek, C.., 1993. Deep crustal structure beneath the central and inner West Carpathians. Tectonophysics, 226: 417-431.

Villa, I.M., 2004. Geochronology of metamorphic rocks. Periodico di Mineralogia, 73: 259-271.

Villa, I.M., Bucher, S., Bousquet, R., Kleinhanns, I.C., Schmid, S.M., 2014. Dating polygenetic metamorphic assemblages along a transect across the Western Alps. Journal of Petrology, 55: 803-830.

Vozár, J., Szalaiová, V., Šantavý, J., 1998. Interpretation of the Western Carpathian deep structures on the basis of gravimetric and seismic sections. In: Geodynamic Development of the Western Carpathians (ed. M. Rakús): 241-257. Geological Survey Slovak Republic, Bratislava.

Vozár, J., Šantavý, J., eds., 1999. Atlas of deep-crustal reflection seismic profiles of the Western Carpathians and their interpretation. Ministry of Environment of Slovak Republic.

Vozárová, A., Konečný, P., Šarinová, K., Vozár, J., 2014. Ordovician and Cretaceous tectonothermal history of the Southern Gemericum Unit from microprobe monazite geochronology (Western Carpathians, Slovakia). International Journal of Earth Sciences (Geologische Rundschau), 103: 1005-1022.

Zane, A., Weiss, Z., 1998. A procedure for classifying rock-forming chlorites based on microprobe data. Rendiconti della Accademia Nazionale dei Lincei, 9: 51-56.

Zaun, P.E., Wagner, G.A., 1985. Fission-track stability in zircons under geological conditions. Nuclear Tracks and Radiation Measurements (1982), 10: 303-307. 